\title{
Un résultat de connexité pour les variétés analytiques $p$-adiques: privilège et noethérianité
}

\author{
Jérôme Poineau
}

\begin{abstract}
Let $k$ be a non-Archimedean field, let $X$ be a $k$-affinoid space and let $f_{1}, \ldots, f_{n}$, with $n \in \mathbb{N}^{*}$, be analytic functions over $X$. If $X$ is irreducible, we prove that the analytic domain $\bigcup_{1 \leqslant j \leqslant n}\left\{x \in X|| f_{j}(x) \mid \geqslant \varepsilon_{j}\right\}$ is still irreducible, provided that $\left(\varepsilon_{1}, \ldots, \varepsilon_{n}\right) \in \mathbb{R}_{+}^{n}$ is small enough. Then, for a general $X$, we precisely describe how the geometric connected components of the spaces $\{x \in X|| f(x) \mid \geqslant \varepsilon\}$ behave with regards to $\varepsilon$. Finally, we obtain a result concerning privileged neighbourhoods and adapt a theorem from complex analytic geometry about Noetherianity for germs of analytic functions.
\end{abstract}

\section{Introduction}

Le travail que nous présentons ici trouve son origine dans une tentative d'adapter au cadre $p$-adique un résultat connu de géométrie analytique complexe. Il s'agit d'un théorème, dû à Frisch, qui assure la noethérianité de l'anneau des germes de fonctions au voisinage de certains compacts. La preuve qui figure dans l'article [Fri67] fait appel, de manière essentielle, à deux notions dont nous ne disposons pas pour des variétés analytiques $p$-adiques: celle de voisinage privilégié et celle de stratification d'une partie semi-analytique.

Cependant, Bănică et Stănăşilă ont abordé le problème de façon légèrement différente et rédigé, dans [BS77, chapitre 5, fin de la section 3], une démonstration, dont les arguments peuvent s'adapter, sans peine. Nous proposons, en appendice à ce texte, un énoncé du théorème, dans le cadre des espaces définis sur un corps ultramétrique complet, accompagné d'une preuve, calquée sur la leur.

La démonstration originale de J. Frisch, bien qu'à présent obsolète, nous a conduit à nous intéresser aux voisinages privilégiés. Nous avons alors cherché à étendre au cadre des variétés $p$-adiques le résultat du Douady (cf. [Dou66, §6, théorème 1]) assurant l'existence de voisinages compacts privilégiés pour les faisceaux cohérents. Ainsi que nous l'expliquerons dans la dernière partie de ce texte, nous pouvons en proposer une démonstration fort simple, pour peu que nous disposions d'une sorte de généralisation du théorème d'extension de Riemann. Elle s'énonce comme suit.

ThÉorème 1. Soit $X$ un espace $k$-affinoïde irréductible et $f_{1}, \ldots, f_{n}$, avec $n \in \mathbb{N}^{*}$, des fonctions analytiques sur $X$. Alors il existe un voisinage $V$ de 0 dans $\mathbb{R}_{+}^{n}$ tel que le domaine analytique de $X$ défini par

$$
V_{\varepsilon}=\bigcup_{1 \leqslant j \leqslant n}\left\{x \in X|| f_{j}(x) \mid \geqslant \varepsilon_{j}\right\}
$$

est irréductible, dès que le $n$-uplet $\varepsilon=\left(\varepsilon_{1}, \ldots, \varepsilon_{n}\right)$ appartient à $V$.

Received 9 June 2006, accepted in final form 9 March 2007, published online 23 January 2008. 2000 Mathematics Subject Classification 14G22.

Keywords: Berkovich spaces, rigid geometry, formal schemes, privilege, noetherianity.

This journal is (C) Foundation Compositio Mathematica 2008. 


\section{J. POINEAU}

Dorénavant, c'est à ce problème que nous consacrerons notre attention. Rappelons que, dans le cadre de la géométrie analytique complexe, le théorème d'extension de Riemann assure, en particulier, qu'un espace irréductible le reste lorsque l'on lui retire un fermé analytique strict. L'analogue de ce théorème pour les variétés analytiques $p$-adiques est également connu de longue date (cf. [Bar70, Lut74]). Dans quelle mesure est-il possible d'ôter un voisinage d'un tel fermé sans nuire à l'irréductibilité?

Remarquons que, sur un ouvert, cadre naturel de la géométrie analytique complexe, toute tentative en ce sens serait vouée à l'échec. Pour nous en convaincre, considérons l'ouvert du plan complexe défini par

$$
U=\left\{z \in \mathbb{C}|| \operatorname{Im}(z) \mid<\frac{1}{|\operatorname{Re}(z)|+1}\right\} .
$$

Alors, dès que $\varepsilon$ est assez petit, le domaine défini par $\{z \in U|| \sin (z) \mid \geqslant \varepsilon\}$ possède une infinité de composantes connexes.

Néanmoins, le problème garde un intérêt pour les espaces analytiques définis sur un corps ultramétrique complet. En effet, les modèles locaux de ces derniers, appelés espaces affinoïdes, se comportent, à bien des égards, comme des espaces compacts. Entre autres propriétés, ils sont quasi-compacts, définis par un nombre fini d'inégalités larges et vérifient un principe du maximum.

Nous nous placerons donc désormais sur un espace affinoïde irréductible $X$ défini sur un corps ultramétrique complet $k$. Notons $\sqrt{\left|k^{*}\right|}$ le Q-espace vectoriel engendré par le groupe des valeurs du corps de base. Dans le cadre de la géométrie analytique rigide, les fonctions prennent leurs valeurs dans l'ensemble, peu ragoûtant, $\sqrt{\left|k^{*}\right|} \cup\{0\}$ et la topologie des espaces est une topologie de Grothendieck, qui n'est guère aisée à manipuler. L'espace $X$ est quasi-compact, mais, en général, nous ne pouvons assurer que l'ensemble $V_{\varepsilon}$ vérifie cette propriété que dans le cas où $\varepsilon \in\left(\sqrt{\left|k^{*}\right|}\right)^{n}$.

Il y a de cela une vingtaine d'années, Berkovich proposa une nouvelle approche des variétés analytiques sur un corps ultramétrique complet (cf. [Berk90] et [Berk93]). La construction qu'il mit en œuvre présentait plusieurs avantages et notamment celui de fournir des espaces possédant de nombreuses propriétés topologiques remarquables: à titre d'exemple, citons la séparation, la compacité et la connexité par arcs locales. Cette dernière propriété nous sera fort utile: le résultat que nous avons en vue imposant à un certain espace d'être connexe, cela nous simplifiera la tâche de pouvoir tracer des chemins.

Ajoutons que, dans ce nouveau cadre, les fonctions prennent leurs valeurs dans un ensemble continu, que l'espace $X$ est compact et qu'il en est de même pour l'espace $V_{\boldsymbol{\varepsilon}}$, quel que soit $\varepsilon \in \mathbb{R}_{+}^{n}$. Ces différentes raisons nous conduisent à nous placer, tout au long de ce texte et sans plus le préciser désormais, dans le cadre des espaces analytiques au sens de Berkovich. Nous y démontrerons le théorème annoncé. Précisons, cependant, que le résultat reste valable dans le cadre de la géométrie analytique rigide.

Restreignons-nous, à présent, au cas d'une seule fonction $f$ sur $X$ et supposons qu'elle soit bornée par 1. Considérons les domaines affinoïdes de $X$ de la forme

$$
V_{\varepsilon}=\{x \in X|| f(x) \mid \geqslant \varepsilon\},
$$

avec $\varepsilon \in[0,1]$. Notre second théorème assure que les composantes connexes des domaines affinoïdes précédents varient de façon modérée en fonction du paramètre $\varepsilon$. Pour le démontrer, nous utiliserons, de manière essentielle, une autre spécificité des espaces construits par Berkovich: dans les bons cas, ils se rétractent sur un sous-ensemble fermé, appelé squelette, qui est muni d'une structure linéaire par morceaux. En particulier, nous parviendrons à lire le paramètre $\varepsilon$ sur un segment réel, homéomorphe à $[0,1]$, tracé sur le disque unité de dimension 1. 


\section{ConneXITÉ EN GÉOMÉTRIQUE ANALYTIQUe $p$-ADIQUE}

Énonçons précisément le théorème en question. Soit $\bar{k}$ une clôture algébrique $k$. Nous noterons $\pi_{0}^{g}$ le foncteur, défini de la catégorie des espaces $k$-analytiques dans celle des ensembles munis d'une action du groupe d'automorphismes $\operatorname{Aut}(\bar{k} / k)$, qui associe à un espace $k$-analytique l'ensemble de ses composantes connexes géométriques.

ThÉorÈme 2. Soient $k$ un corps ultramétrique complet, $X$ un espace $k$-affinoïde et $f$ une fonction analytique sur $X$. Alors il existe une partition finie $\mathcal{P}$ de $\mathbb{R}^{+}$de la forme

$$
\left.\left.\left.\left.\mathcal{P}=\left\{\left[0, a_{0}\right],\right] a_{0}, a_{1}\right], \ldots,\right] a_{r-1}, a_{r}\right],\right] a_{r},+\infty[\},
$$

où $r \in \mathbb{N}$ et $\left(a_{i}\right)_{0 \leqslant i \leqslant r}$ est une suite croissante d'éléments de $R_{X} \cup\{0\}$, satisfaisant la condition suivante: quel que soit $I \in \mathcal{P}$, quels que soient $\varepsilon^{\prime}, \varepsilon \in I$, avec $\varepsilon^{\prime} \leqslant \varepsilon$, l'inclusion

$$
\{x \in X|| f(x) \mid \geqslant \varepsilon\} \subset\left\{x \in X|| f(x) \mid \geqslant \varepsilon^{\prime}\right\}
$$

induit une bijection

$$
\pi_{0}^{g}(\{x \in X|| f(x) \mid \geqslant \varepsilon\}) \rightarrow \pi_{0}^{g}\left(\left\{x \in X|| f(x) \mid \geqslant \varepsilon^{\prime}\right\}\right) .
$$

Le même résultat vaut pour le foncteur qui associe à un espace $k$-analytique le Aut $(\bar{k} / k)$-ensemble de ses composantes irréductibles géométriques.

Dans ce théorème, l'ensemble $R_{X}$ désigne le sous-Q-espace vectoriel de $\mathbb{R}_{+}^{*}$ engendré par les valeurs non nulles de la norme spectrale sur l'algèbre de $X$. Par exemple, si l'espace $X$ est strictement $k$-affinoïde, on a $R_{X}=\sqrt{\left|k^{*}\right|}$.

Remarquons que, dans le cadre de la géométrie rigide, Abbes et Saito (cf. [AS02, 5.1]) ont déjà démontré ce dernier résultat pour un intervalle du type $[a,+\infty$ [, avec $a>0$, et en ne s'intéressant qu'au cardinal de l'ensemble des composantes connexes géométriques. Nous signalons également que, dans leur article, les bornes des intervalles sont interprétés comme les sauts d'une certaine filtration de ramification.

La démonstration que nous proposons s'effectuera en quatre étapes, correspondant aux quatre premières parties de ce texte. Dans la section 1, nous étudierons la manière dont varient les composantes géométriquement connexes des fibres d'un morphisme entre espaces affinoïdes. Lorsqu'elles se réaliseront comme composantes connexes, nous chercherons à les repérer par des sections. Des questions proches ont déjà été traitées pour des morphismes entre schémas: nous savons, par exemple, d'après [Gro66, 9.7.9], que, pour un morphisme de présentation finie, la fonction qui a un point de la base associe le nombre géométrique de composantes connexes de sa fibre est localement constructible sur la base. Nous parviendrons à nos fins en appliquant des résultats de ce type sur la fibre spéciale d'un modèle formel, judicieusement choisi, du morphisme entre espaces affinoïdes dont nous sommes partis.

La difficulté principale tient dans la démonstration de l'existence d'un modèle possédant de bonnes propriétés. Elle nous est assurée par le théorème de la fibre réduite (cf. [Bos95b]), pourvu que le morphisme entre espaces affinoïdes soit plat et à fibres géométriquement réduites. Dans ce cas, nous parviendrons à exhiber une partition finie de la base en domaines analytiques sur lesquels le nombre géométrique de composantes connexes des fibres est constant.

Les sections 2 et 3 seront consacrées aux domaines définis par

$$
\{x \in X|| f(x) \mid \geqslant \varepsilon\},
$$

pour $\varepsilon>0$, dans le cas particulier d'un espace $X$ strictement affinoïde intègre défini sur un corps algébriquement clos et d'une fonction $f$ de norme spectrale égale à 1 . Nous montrerons que la variation de leurs composantes connexes, en fonction de $\varepsilon$, est liée à un problème du type précédent. $\grave{A}$ cet effet, nous construirons explicitement un morphisme $\tau$ au-dessus du disque analytique $\mathbf{D}=$ $\mathscr{M}(k\{U\})$ de dimension 1 dont les fibres seront isomorphes, après extension du corps de base, 


\section{J. POINEAU}

aux domaines affinoïdes en question. Le paramètre réel $\varepsilon$ recevra, lui aussi, une interprétation géométrique en termes de valeur absolue de l'évaluation de la fonction $U$ sur le disque.

Afin d'appliquer les résultats du début, nous devrons nous assurer que le morphisme $\tau$ vérifie certaines propriétés. Nous démontrerons sans peine qu'il est plat, mais buterons sur le caractère géométriquement réduit de l'une des fibres. Dans la section 3 nous modifierons le morphisme $\tau$ de façon à passer outre ce problème. Les techniques mises en jeu relèveront, cette fois-ci, de la géométrie algébrique, puisque nous travaillerons sur des spectres, au sens schématique, d'algèbres affinoïdes. L'argument principal que nous utiliserons sera le théorème d'élimination de la ramification démontré par Epp dans [Epp73]. Par ce biais, nous parviendrons à obtenir des informations sur les fibres de $\tau$ voisines de celle qui présente des multiplicités et à ramener le problème de la connexité de $\{x \in X|| f(x) \mid \geqslant \varepsilon\}$, pour $\varepsilon$ proche de 0 , à celui de $\{x \in X|| f(x) \mid>0\}$. Nous concluerons grâce à l'analogue ultramétrique du théorème de Hartogs (cf. [Bar70] ou [Lut74]).

Dans la section 4 nous expliquerons comment déduire les théorèmes 1 et 2 en toute généralité, à partir des cas particuliers considérés dans les parties précédentes.

\section{Connexité des fibres d'un morphisme}

Dans cette partie, nous fixerons un corps ultramétrique complet $k$ dont nous supposerons que la valuation n'est pas triviale. Nous noterons $k^{\circ}$ son anneau de valuation et $\tilde{k}$ son corps résiduel.

Soit $\mathscr{B}$ une algèbre strictement $k$-affinoïde. Rappelons qu'il existe deux façons de réduire l'espace affinoïde $\mathscr{M}(\mathscr{B})$ en une variété algébrique. La première, que l'on trouvera expliquée, par exemple, dans [Berk90, 2.4], utilise la semi-norme spectrale, notée $|\cdot|_{\text {sup }}$, sur l'algèbre strictement $k$-affinoïde $\mathscr{B}$. Elle associe à l'espace affinoïde $\mathscr{M}(\mathscr{B})$ la variété algébrique $\operatorname{Spec}(\tilde{\mathscr{B}})$ où $\tilde{\mathscr{B}}$ désigne le quotient de l'anneau $\mathscr{B}^{\circ}=\left\{g \in \mathscr{B} /|g|_{\text {sup }} \leqslant 1\right\}$ par l'idéal $\mathscr{B}^{\circ \circ}=\left\{g \in \mathscr{B} /|g|_{\text {sup }}<1\right\}$. Dans ce cas, l'application de réduction est surjective, anticontinue et induit une bijection entre les composantes connexes.

La seconde réduction, due à Raynaud (cf. [Ray74]), consiste à interpréter l'espace affinoïde comme la fibre générique d'un schéma formel plat sur un anneau de valuation. La variété algébrique associée est alors définie comme la fibre spéciale du modèle. On démontre aisément que tout espace affinoïde admet un modèle formel. Un résultat plus difficile assure même que tout morphisme peut se réaliser comme un morphisme entre modèles, ce dernier pouvant être choisi plat lorsque le morphisme de départ l'est. Pour plus de détails, nous renvoyons aux articles de référence [BL93a] et [BL93b].

Rappelons qu'un $k^{\circ}$-schéma formel est dit admissible s'il est localement topologiquement de présentation finie et plat $\operatorname{sur} \operatorname{Spf}\left(k^{\circ}\right)$ (i.e. sans $k^{\circ}$-torsion). Nous dirons qu'un morphisme entre espaces $k$-affinoïdes $\mathscr{M}(\mathscr{C}) \rightarrow \mathscr{M}(\mathscr{B})$ est plat lorsque le morphisme associé $\mathscr{B} \rightarrow \mathscr{C}$ entre algèbres $k$-affinoïdes l'est.

Lemme 1.1. Tout morphisme plat entre $k^{\circ}$-schémas formels admissibles le reste après changement de base par un morphisme entre $k^{\circ}$-schémas formels admissibles et après extension des scalaires de $k^{\circ}$ à $L^{\circ}$, où $L^{\circ}$ désigne l'anneau de valuation d'une extension ultramétrique complète $L$ de $k$.

Ces résultats restent valables pour les morphismes entre espaces strictement $k$-affinoïdes.

Démonstration. Notons $m$ l'idéal maximal de $k^{\circ}$. Soit $\varphi: A \rightarrow B$ un morphisme de $k^{\circ}$-algèbres topologiquement de présentation finie. D'après [BL93a, 1.6], le morphisme $\varphi$ est plat si, et seulement si, quel que soit $n \in \mathbb{N}^{*}$, le morphisme induit

$$
A \otimes_{k^{\circ}}\left(k^{\circ} / m^{n}\right) \rightarrow B \otimes_{k^{\circ}}\left(k^{\circ} / m^{n}\right)
$$

est plat. Le résultat pour les schémas formels en découle immédiatement. Celui pour les espaces strictement $k$-affinoïdes s'y ramène puisqu'un morphisme plat entre tels espaces admet un modèle plat, d'après [BL93b, 5.10]. 


\section{ConneXITÉ EN GÉOMÉTRIQUE ANALYTIQUe $p$-ADIQUE}

Les deux lemmes suivants illustrent l'importance des morphismes plats entre modèles formels. Lorsque, par la suite, nous considérerons un $k^{\circ}$-schéma formel admissible, nous désignerons sa fibre générique (respectivement spéciale) par le même symbole, auquel nous ajouterons un $\eta$ (respectivement une $s$ ) en indice. Nous adopterons la même convention pour les morphismes entre tels objets.

Lemme 1.2. Sur un schéma formel admissible, l'application de réduction est surjective.

Démonstration. Soit $\mathscr{Y}$ un $k^{\circ}$-schéma formel admissible. Nous pouvons le supposer affine, d'algèbre $\mathscr{B}$. Soit $\tilde{y}$ un point de la fibre spéciale $\mathscr{Y}_{s}$ de $\mathscr{Y}$. Notons $\tilde{k}(\tilde{y})$ son corps résiduel. Puisque $\mathscr{Y}_{s}$ est un $\tilde{k}$-schéma de type fini, il existe une base de transcendance $\left(T_{1}, \ldots, T_{r}\right)$, avec $r \in \mathbb{N}$, de $\tilde{k}(\tilde{y})$ sur $\tilde{k}$.

Soit $K$ le complété du corps $k\left(U_{1}, \ldots, U_{r}\right)$ pour la norme de Gauß. Son corps résiduel est isomorphe à $\tilde{k}\left(T_{1}, \ldots, T_{r}\right)$. Considérons le complété $L$ d'une clôture algébrique de $K$. D'après [BGR84, $3.4 .1 / 5]$, son corps résiduel $\tilde{L}$ est une clôture algébrique de $\tilde{k}\left(T_{1}, \ldots, T_{r}\right)$ et contient donc un corps isomorphe à $\tilde{k}(\tilde{y})$.

D'après le lemme 1.1, le morphisme

$$
\varphi: \mathscr{Z}=\mathscr{Y} \times{ }_{\operatorname{Spf}\left(k^{\circ}\right)} \operatorname{Spf}\left(L^{\circ}\right) \rightarrow \operatorname{Spf}\left(L^{\circ}\right)
$$

est plat, autrement dit, $\mathscr{Z}$ définit un $L^{\circ}$ - schéma formel admissible. Par construction, la fibre $\varphi_{s}^{-1}(\tilde{y})$ au-dessus de $\tilde{y}$ possède un point fermé de corps résiduel isomorphe à $\tilde{L}$. Ce point est encore fermé dans $\mathscr{Z}_{s}$. D'après [Bert96, 1.1.5], c'est l'image d'un point de la fibre générique, ce qui permet de conclure.

De ce résultat, déduisons-en un autre, que nous utiliserons à de nombreuses reprises.

Lemme 1.3. Soit $\varphi: \mathscr{Y} \rightarrow \mathscr{Z}$ un morphisme de $k^{\circ}$-schémas formels admissibles. Nous noterons indifféremment $\pi$ les deux morphismes de spécialisation.

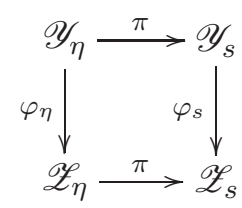

Si le morphisme $\varphi$ est plat, alors les spécialisations des fibres de $\varphi_{\eta}$ coïncident avec les fibres du morphisme spécialisé $\varphi_{s}$. Autrement dit, quel que soit $z \in \mathscr{Z}_{\eta}$, on dispose d'un isomorphisme

$$
\pi\left(\varphi_{\eta}^{-1}(z)\right) \simeq \varphi_{s}^{-1}(\pi(z)) .
$$

Démonstration. Seule la surjectivité nécessite une démonstration. Nous pouvons supposer que le schéma formel $\mathscr{Z}$ est affine, d'algèbre $\mathscr{C}$. Soit $z$ un point de $\mathscr{Z}_{\eta}$. Il lui correspond un caractère $\chi_{z}: \mathscr{C} \otimes_{k^{\circ}} k \rightarrow \mathscr{H}(z)$. On en déduit un morphisme $\chi: \mathscr{C} \rightarrow \mathscr{H}(z)^{\circ}$, la notation $\mathscr{H}(z)^{\circ}$ désignant l'anneau de valuation du corps ultramétrique complet $\mathscr{H}(z)$.

Par le même raisonnement que dans le lemme précédent, on démontre que le schéma formel $\mathscr{Y} \times \mathscr{Z} \operatorname{Spf}\left(\mathscr{H}(z)^{\circ}\right)$ est un $\mathscr{H}(z)^{\circ}$-schéma formel admissible. Ses fibres générique et spéciale sont respectivement isomorphes à $\varphi_{\eta}^{-1}(z)$ et $\varphi_{s}^{-1}(\pi(z)) \otimes_{\tilde{k}} \tilde{\mathscr{H}}(z)$. Le morphisme $\varphi_{s}^{-1}(\pi(z)) \otimes_{\tilde{k}} \tilde{\mathscr{H}}(z) \rightarrow$ $\varphi_{s}^{-1}(\pi(z))$ est surjectif et le lemme précédent nous permet de conclure.

Des liens, détaillés dans [Bos95b, § 1], existent parfois entre la réduction définie par la norme spectrale et celle au sens de Raynaud. Citons qu'elles coïncident lorsque la fibre spéciale du modèle formel est réduite. La prochaine proposition découlera de ce résultat. Énonçons, au préalable, quelques définitions. 


\section{J. POINEAU}

DÉfinition 1.4. Soit $p: A \rightarrow B$ une application continue entre espaces topologiques. Soient un entier $r \in \mathbb{N}$, une partie $P$ de $B$, une famille d'espaces topologiques $\left(Q_{i}\right)_{1 \leqslant i \leqslant r}$ et deux familles d'applications continues

$$
\mathbf{s}=\left(s_{i}: Q_{i} \rightarrow B\right)_{1 \leqslant i \leqslant r} \quad \text { et } \quad \mathbf{t}=\left(t_{i}: Q_{i} \rightarrow A\right)_{1 \leqslant i \leqslant r}
$$

Nous dirons que les familles $\mathbf{s}$ et $\mathbf{t}$ repèrent les composantes connexes des fibres de $p$ au-dessus de $P$ si les conditions suivantes sont satisfaites:

(a) quel que soit $i \in \llbracket 1, r \rrbracket$, l'image de l'application $s_{i}$ recouvre $P$;

(b) quel que soit $b \in P$, la fibre $p^{-1}(b)$ possède exactement $r$ composantes connexes;

(c) quels que soient $b \in P$ et la composante connexe $C$ de $p^{-1}(b)$, il existe un unique $i \in \llbracket 1, r \rrbracket$ pour lequel on ait

$$
\forall b^{\prime} \in s_{i}^{-1}(b), \quad t_{i}\left(b^{\prime}\right) \in C .
$$

DÉfinition 1.5. Soient $\varphi: Y \rightarrow Z$ un morphisme de schémas et $P$ une partie de l'espace topologique sous-jacent à $Z$. Nous dirons que le morphisme $\varphi$ admet un découpage au-dessus de $P$ s'il existe un entier $r \in \mathbb{N}$ et des familles finies

$$
\mathbf{s}=\left(s_{i}: Z_{i} \rightarrow Z\right)_{1 \leqslant i \leqslant r} \quad \text { et } \quad \mathbf{t}=\left(t_{i}: Z_{i} \rightarrow Z_{i} \times{ }_{Z} Y\right)_{1 \leqslant i \leqslant r}
$$

de morphismes entre schémas vérifiant les conditions suivantes:

(a) quel que soit $i \in \llbracket 1, r \rrbracket$, le morphisme $t_{i}$ définit une section du morphisme $Z_{i} \times{ }_{Z} Y \rightarrow Z_{i}$, obtenu à partir de $\varphi$ par le changement de base $s_{i}$;

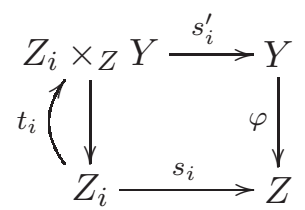

(b) quel que soit $i \in \llbracket 1, r \rrbracket$, le morphisme $s_{i}$ est étale;

(c) les familles $\mathbf{s}$ et $\mathbf{t}^{\prime}=\left(s_{i}^{\prime} \circ t_{i}\right)_{1 \leqslant i \leqslant r}$ repèrent les composantes connexes des fibres de $\varphi$ au-dessus de $P$.

Nous adoptons la même définition pour un morphisme entre espaces $k$-analytiques en remplaçant les morphismes étales par des morphismes quasi-étales ${ }^{1}$ dont la source est un espace $k$-analytique compact.

DÉfinition 1.6. Nous dirons qu'un schéma (respectivement espace $k$-analytique) est déployé lorsque ses composantes connexes sont géométriquement connexes.

Proposition 1.7. Soit $\varphi: \mathscr{Y} \rightarrow \mathscr{Z}$ un morphisme plat entre $k^{\circ}$-schémas formels admissibles et quasi-compacts. Supposons que le morphisme $\varphi_{s}$, induit entre les fibres spéciales, soit surjectif et que ses fibres soient géométriquement réduites et déployées. Alors il existe une partition finie $\mathcal{P}$ de $\mathscr{Z}_{s}$ vérifiant les conditions suivantes:

(a) les éléments de $\mathcal{P}$ sont des parties constructibles de $\mathscr{Z}_{s}$;

(b) quel que soit $P \in \mathcal{P}$, le morphisme $\varphi_{\eta}$ admet un découpage au-dessus du tube de $P$.

Démonstration. Intéressons-nous au morphisme $\varphi_{s}: \mathscr{Y}_{s} \rightarrow \mathscr{Z}_{s}$ entre schémas de type fini sur le corps $\tilde{k}$. Soit $Z$ un fermé irréductible de $\mathscr{Z}_{s}$ de point générique $\zeta$. Notons

$$
\psi: Y=Z \times \mathscr{Z}_{s} \mathscr{Y}_{s} \rightarrow Z
$$

\footnotetext{
${ }^{1}$ Les morphismes quasi-étales définis par Berkovich (cf. [Berk94, §3]) correspondent aux morphismes rig-étales de la géométrie rigide (cf. [Bos95a, 3.1]).
} 


\section{ConneXITÉ EN GÉOMÉTRIQUE ANALYTIQUe $p$-ADIQUE}

le morphisme induit par $\varphi_{s}$ au-dessus de $Z$. Soient $C_{1}, \ldots, C_{r}$, avec $r \in \mathbb{N}$, les composantes connexes de la fibre générique $\psi^{-1}(\zeta)$. Pour chaque $i \in \llbracket 1, r \rrbracket$, choisissons un voisinage ouvert $U_{i}$ de $C_{i}$ dans $Y$ dont la trace sur $\psi^{-1}(\zeta)$ soit égale à $C_{i}$. L'ensemble des points de $Y$ appartenant à au moins deux de ces voisinages forme une partie constructible donc, d'après le théorème de Chevalley (cf. [Gro64, 1.8.4]), son image $V$ définit une partie constructible de $Z$. Puisque la partie $V$ ne contient pas le point générique $\zeta$, elle évite même un ouvert $W$ autour de ce point.

Une nouvelle utilisation du théorème de Chevalley nous montre qu'il existe un voisinage ouvert $W^{\prime}$ de $\zeta$ dans $W$ tel que, quel que soit $z \in W^{\prime}$, les traces des ouverts $U_{i}$, avec $i \in \llbracket 1, r \rrbracket$, recouvrent la fibre $\psi^{-1}(z)$. Pour $z \in W^{\prime}$, elles sont donc réunions de composantes connexes disjointes de $\psi^{-1}(z)$. D'après [Gro66, 9.7.8], le nombre géométrique de composantes connexes des fibres de $\psi$ est constant sur un voisinage ouvert $W^{\prime \prime}$ de $\zeta$ dans $W^{\prime}$. Pour $i \in \llbracket 1, r \rrbracket$, notons $V_{i}$ le voisinage ouvert de $C_{i}$ défini par $V_{i}=U_{i} \cap \psi^{-1}\left(W^{\prime \prime}\right)$. Puisque les fibres de $\varphi_{s}$ sont déployées, quel que soit $z \in W^{\prime \prime}$, les traces des ouverts $V_{i}$, pour $i \in \llbracket 1, r \rrbracket$, sur la fibre $\psi^{-1}(z) \simeq \varphi_{s}^{-1}(z)$ sont exactement les composantes connexes de cette fibre.

Soit $i \in \llbracket 1, r \rrbracket$. Choisissons un ouvert $V_{i}^{\prime}$ de $\mathscr{Y}_{s}$ dont la trace sur $Y$ soit égale à $V_{i}$. Notons $\varphi_{i}: V_{i}^{\prime} \rightarrow \mathscr{Z}_{s}$ le morphisme induit par $\varphi_{s}$ sur $V_{i}^{\prime}$. Par hypothèse, la fibre

$$
\varphi_{i}^{-1}(\zeta) \simeq C_{i}
$$

est géométriquement réduite et non vide. Elle contient donc un point $y_{i}$ en lequel elle est lisse. Puisque le morphisme $\varphi_{s}$ est plat, ce point est encore lisse dans $\mathscr{Y}_{s}$. Quitte à restreindre $V_{i}^{\prime}$, nous pouvons donc supposer que $\varphi_{i}$ est lisse. On en déduit qu'il existe un schéma quasi-compact $S_{i}$, un morphisme étale $s_{i}: S_{i} \rightarrow \mathscr{Z}_{s}$ et un point $\zeta_{i}^{\prime}$ au-dessus de $\zeta$ tel que le morphisme $S_{i} \times \mathscr{Z}_{s} V_{i}^{\prime} \rightarrow S_{i}$, obtenu à partir de $\varphi_{i}$ par le changement de base $s_{i}$, admette une section $t: S_{i} \rightarrow S_{i} \times \mathscr{Z}_{s} V_{i}^{\prime}$, où $t\left(\zeta_{i}^{\prime}\right)$ s'envoie sur $y_{i}$.

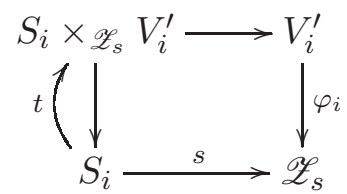

Les images des morphismes étales $s_{i}$, pour $i \in \llbracket 1, r \rrbracket$, contiennent un voisinage ouvert commun de $\zeta$ dans $Z$. Par construction, le morphisme $\varphi_{s}$ y admet un découpage. Un argument de récurrence noethérienne nous montre ensuite qu'il existe une partition $\mathcal{P}$ de $\mathscr{Z}_{s}$ en parties constructibles audessus desquelles le morphisme $\varphi_{s}$ admet un découpage.

Remarquons que nous pouvons relever les constructions précédentes aux schémas formels. Considérons, en effet, une restriction $\psi$ de $\varphi_{s}$ à un ouvert lisse $U$ et un morphisme étale $s: S \rightarrow \mathscr{Z}_{s}$ tel que le morphisme $S \times \mathscr{Z}_{s} U \rightarrow S$, obtenu à partir de $\psi$ par le changement de base $s$, admette une section $t$. Notons $\mathscr{U}$ le sous-schéma formel ouvert de $\mathscr{Y}$ dont l'espace topologique sous-jacent est l'ouvert $U$. D'après [Berk94, 2.1], le morphisme $s$ admet un modèle formel étale $\mathscr{S} \rightarrow \mathscr{Z}$. D'autre part, puisque le morphisme $\mathscr{S} \times \mathscr{Z} \mathscr{U} \rightarrow \mathscr{S}$ est lisse, la propriété de relèvement infinitésimal nous assure que la section $t$ se relève en une section formelle $T$.

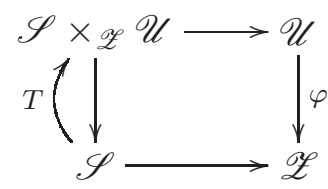

Expliquons, à présent, comment passer des schémas formels à leur fibre générique. Soient $z$ un point de $\mathscr{Z}_{\eta}$ et $\tilde{z}$ son image dans $\mathscr{Z}_{s}$ par l'application de spécialisation. Puisque le morphisme $\varphi$ est plat et à fibres géométriquement réduites, la réduction de $\varphi_{\eta}^{-1}(z)$ obtenue par la norme spectrale coïncide avec celle au sens des modèles, à savoir la fibre $\varphi_{s}^{-1}(\tilde{z})$. D'après les propriétés de 


\section{J. Poineau}

la réduction par la norme spectrale, chaque composante connexe de la fibre analytique est le tube d'une composante connexe de sa réduction, et vice versa.

D'autre part, si $\mathscr{S} \rightarrow \mathscr{Z}$ est un morphisme étale entre schémas formels quasi-compacts, alors le morphisme $\mathscr{S}_{\eta} \rightarrow \mathscr{Z}_{\eta}$, induit entre les fibres génériques, est un morphisme quasi-étale entre espaces $k$-analytiques compacts.

Le théorème suivant concerne le comportement des composantes connexes des fibres d'un morphisme entre espaces analytiques. La démonstration repose sur le théorème de la fibre réduite (cf. [Bos95b]) qui nous permet de nous ramener à un modèle du morphisme satisfaisant les hypothèses de la proposition précédente. Cette fois encore, nous commençons par poser une définition.

DÉfinition 1.8. Soit $Y$ un espace $k$-affinoïde. Une partie $V$ de $Y$ est dite simple si elle peut s'obtenir par combinaison booléenne finie de domaines affinoïdes du type

$$
\{z \in Y|| h(z) \mid=1\}
$$

où $h$ désigne une fonction analytique sur $Y$ de norme spectrale égale à 1 .

Remarquons, dès à présent, qu'une partie simple d'un espace $k$-affinoïde est voisinage de chacun de ses points rigides. En effet, elle s'obtient par réunion et intersection d'un nombre fini de parties qui sont soit des domaines affinoïdes, soit des ouverts et pour lesquelles ce résultat est vrai.

ThÉORÈme 1.9. Soit $\varphi: Y \rightarrow Z$ un morphisme plat et surjectif entre espaces strictement $k$-affinoïdes dont les fibres soient géométriquement réduites et déployées. Alors il existe une partition finie $\mathcal{P}$ de $Z$ vérifiant les conditions suivantes:

(a) les éléments de $\mathcal{P}$ sont des parties simples de domaines affinö̈des de $Z$;

(b) quel que soit $P \in \mathcal{P}$, le morphisme $\varphi$ admet un découpage au-dessus de $P$.

Démonstration. Les hypothèses de l'énoncé nous permettent d'appliquer le théorème de la fibre réduite. Celui-ci nous assure qu'il existe un diagramme commutatif de schémas formels

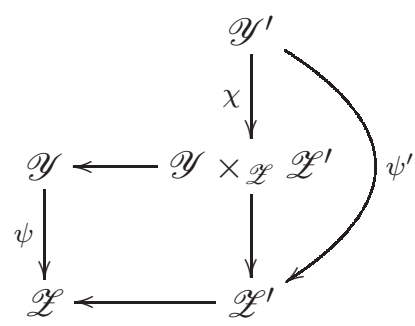

où :

(a) le morphisme $\psi: \mathscr{Y} \rightarrow \mathscr{Z}$ induit le morphisme $\varphi: Y \rightarrow Z$ par passage aux fibres génériques;

(b) le morphisme $Z^{\prime} \rightarrow Z$, où $Z^{\prime}$ désigne la fibre générique de $\mathscr{Z}^{\prime}$, est quasi-étale et surjectif;

(c) le morphisme $\chi: \mathscr{Y}^{\prime} \rightarrow \mathscr{Y} \times \mathscr{Z}_{\mathscr{Z}^{\prime}}$ est fini et induit un isomorphisme entre les fibres génériques ;

(d) le morphisme $\psi^{\prime}: \mathscr{Y}^{\prime} \rightarrow \mathscr{Z}^{\prime}$ est plat et ses fibres sont géométriquement réduites.

Vérifions que nous pouvons appliquer la proposition précédente au morphisme $\psi^{\prime}$. Les $k^{\circ}$-schémas formels obtenus comme modèles des espaces analytiques sont bien admissibles et quasi-compacts, en vertu de l'équivalence de catégories [BL93a, 4.1]. Tous les schémas formels que nous considérerons sont également de ce type.

Les fibres de $\psi_{\eta}^{\prime}$ sont isomorphes, après extension du corps de base, à des fibres de $\psi_{\eta}=\varphi$. Par hypothèse, le morphisme $\varphi$ est surjectif. Il en est donc de même pour $\psi_{\eta}^{\prime}$, puis pour $\psi_{s}^{\prime}$, d'après le lemme 1.3. 


\section{ConneXITÉ EN GÉOMÉTRIQUE ANALYTIQUe $p$-ADIQUE}

Il nous reste à démontrer que les fibres de $\psi^{\prime}$ sont déployées. Puisque le morphisme $\chi$ est fini, il suffit même de le vérifier sur le morphisme $\mathscr{Y} \times \mathscr{Z}_{\mathscr{Z}^{\prime}} \rightarrow \mathscr{Z}^{\prime}$, autrement dit sur le morphisme $\psi$. Or le morphisme $\psi$ est plat et à fibres géométriquement réduites, donc le nombre de composantes connexes (respectivement géométriquement connexes) des fibres de $\psi_{\eta}$ est identique à celui de leur réduction. D'après le lemme 1.3, toutes les fibres de $\psi_{s}$ peuvent être obtenues par de telles réductions. Elles sont déployées, puisque les fibres de $\varphi$ le sont, par hypothèse.

D'après la proposition 1.7, il existe une partition finie $\mathcal{P}$ de $\mathscr{Z}_{s}^{\prime}$ en parties constructibles audessus des tubes desquelles le morphisme $\psi_{\eta}^{\prime}$ admet un découpage. Notons $\mathcal{Q}$ l'ensemble de ces tubes. Puisque le morphisme $\chi$ induit un isomorphisme entre les fibres génériques et que le morphisme $\lambda: Z^{\prime} \rightarrow Z$ est quasi-étale, le morphisme $\varphi=\psi_{\eta}$ admet un découpage au-dessus de l'image par $\lambda$ de toute partie de $\mathcal{Q}$. Puisque le morphisme $Z^{\prime} \rightarrow Z$ est surjectif, nous démontrons ainsi l'existence d'un recouvrement fini de $Z$ par des parties au-dessus desquelles le morphisme $\varphi$ admet un découpage. Il est aisé d'en déduire une partition de $Z$ vérifiant la même propriété et composée uniquement de combinaisons booléennes des parties précédentes.

Pour clore la démonstration, il nous reste à vérifier que l'image par $\lambda$ de tout élément de $\mathcal{Q}$ est de la forme désirée. Soit $Q \in \mathscr{Z}_{\eta}^{\prime}$ un élément de $\mathcal{Q}$. Il est obtenu comme le tube d'une partie constructible $P$ de $\mathscr{Z}_{s}^{\prime}$. Le morphisme $\lambda: Z^{\prime} \rightarrow Z$ est plat donc, d'après [BL93b, 5.2], il existe un diagramme commutatif de schémas formels

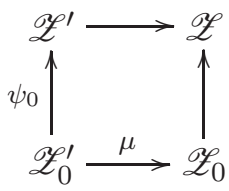

où le morphisme $\mu: \mathscr{Z}_{0}^{\prime} \rightarrow \mathscr{Z}_{0}$ est plat et induit encore le morphisme $\lambda: Z^{\prime} \rightarrow Z$ entre les fibres génériques.

Le tube $Q \subset Z^{\prime}$ de $P$ est identique à celui de $\left(\psi_{0}\right)_{s}^{-1}(P)$. Le morphisme $\mu_{s}:\left(\mathscr{Z}_{0}^{\prime}\right)_{s} \rightarrow\left(\mathscr{Z}_{0}\right)_{s}$ induit par $\mu$ est de type fini et envoie donc la partie constructible $\left(\psi_{0}\right)_{s}^{-1}(P)$ sur une partie constructible $P_{0}$ de $\left(\mathscr{Z}_{0}\right)_{s}$, d'après le théorème de Chevalley. Puisque le morphisme $\mu$ est plat, d'après le lemme 1.3, le tube de $P_{0}$ dans $Z$ n'est autre que l'image de $Q$ dans $Z$ par $\lambda$.

Il nous reste, désormais, à montrer que le tube d'une partie constructible de $\mathscr{Z}_{s}$ est une réunion finie de parties simples de domaines affinoïdes de $\mathscr{Z}_{\eta}$. Ce résultat provient directement des propriétés de la réduction lorsque le schéma formel considéré est affine. Nous concluons en remarquant que le schéma formel quasi-compact $\mathscr{Z}$ admet un recouvrement fini par de tels ouverts.

Rappelons, maintenant, que le nombre géométrique de composantes connexes d'un espace affinoïde ne change pas lorsque l'on étend le corps de base. On trouvera la démonstration de ce résultat dans [Duc03a, théorème 5.5]. On pourrait également le déduire des théorèmes d'extension du corps de base pour les faisceaux étales qui figurent dans [Berk93].

Par un raisonnement très proche de celui que nous venons de mettre en œuvre, nous obtenons le théorème suivant.

ThÉorème 1.10. Soit $\varphi: Y \rightarrow Z$ un morphisme plat entre espaces strictement $k$-affinoïdes dont les fibres soient géométriquement réduites. Alors il existe une partition finie de $Z$ en parties simples de domaines affinoïdes au-dessus desquelles le nombre géométrique de composantes connexes des fibres est constant. En particulier, ce nombre est constant au voisinage des points rigides.

Démonstration. Appliquons le théorème de la fibre réduite et reprenons les notations de la preuve précédente. Le morphisme $\psi_{s}^{\prime}: \mathscr{Y}_{s}^{\prime} \rightarrow \mathscr{Z}_{s}^{\prime}$ est un morphisme de type fini entre deux variétés algébriques de type fini sur $\tilde{k}$. D'après [Gro66, 9.7.9], il existe donc une partition finie $\mathcal{P}$ de $\mathscr{Z}_{s}^{\prime}$ 


\section{J. POINEAU}

en parties constructibles au-dessus desquelles le nombre géométrique de composantes connexes des fibres de $\psi_{s}^{\prime}$ soit constant.

Soit $P \in \mathcal{P}$. Notons $Q \subset Z^{\prime}$ le tube de $P$. Puisque le morphisme $\psi^{\prime}$ est plat et que ses fibres sont géométriquement réduites, le nombre géométrique de composantes connexes des fibres de $\psi_{\eta}^{\prime}$ est constant au-dessus de tout point de $Q$. On en déduit le même résultat pour le morphisme $\psi$ au-dessus de tout point de $\lambda(Q)$. Par un raisonnement en tout point identique à celui exposé dans la preuve précédente, nous obtenons une partition de $Z$ en parties simples de domaines affinoïdes jouissant des mêmes propriétés.

\section{Loin de l'hypersurface}

Consacrons-nous, tout d'abord, à la démonstration du théorème 2, dans un cas particulier. Dans toute cette partie, $k$ désignera un corps algébriquement clos dont la valuation n'est pas triviale, $X$ un espace strictement $k$-affinoïde intègre d'algèbre $\mathscr{A}$ et $f$ une fonction analytique sur $X$ de norme spectrale égale à 1 . Pour $\varepsilon>0$, définissons le domaine affinoïde $V_{\varepsilon}$ de $X$ par

$$
V_{\varepsilon}=\{x \in X /|f(x)| \geqslant \varepsilon\} .
$$

Nous ne nous intéresserons, pour l'instant, qu'aux composantes connexes des espaces $V_{\varepsilon}$, avec $\varepsilon>0$.

Tâchons, tout d'abord, de remplacer le paramètre réel $\varepsilon$ par un autre que nous saurons interpréter géométriquement. Pour ce faire, plaçons-nous au-dessus du disque de dimension 1 et de rayon 1 défini par $\mathbf{D}=\mathscr{M}(k\{U\})$. Dans la suite, nous noterons simplement 0 le point rigide de $\mathbf{D}$ défini par l'équation $U=0$. L'injection $k\{U\} \hookrightarrow \mathscr{A}\{T, U\}$ induit un morphisme $t: k\{U\} \rightarrow$ $\mathscr{A}\{T, U\} /(f T-U)$. Nous noterons $\tau$ le morphisme correspondant entre espaces affinoïdes. L'algèbre $k$-affinoïde $\mathscr{A}\{T, U\} /(f T-U)$ hérite de nombreuses propriétés de l'algèbre $k$-affinoïde $\mathscr{A}$. Le lemme suivant en fournit un exemple.

LEMme 2.1. Les algèbres $k$-affinoïdes $\mathscr{A}\{T, U\} /(f T-U)$ et $\mathscr{A}\{T\}$ sont isomorphes. En particulier, l'algèbre $\mathscr{A}\{T, U\} /(f T-U)$ est intègre. En outre, elle est intégralement close lorsque $\mathscr{A}$ l'est.

Démonstration. On vérifie sans peine que le morphisme de $\mathscr{A}$-algèbres

$$
s: \mathscr{A}\{T, U\} /(f T-U) \rightarrow \mathscr{A}\{T\}
$$

défini par $s(T)=T$ et $s(U)=f T$ est un isomorphisme dont l'inverse est le morphisme de $\mathscr{A}$-algèbres

$$
s^{-1}: \mathscr{A}\{T\} \rightarrow \mathscr{A}\{T, U\} /(f T-U)
$$

défini par $s^{-1}(T)=T$.

Supposons, à présent, que $\mathscr{A}$ soit intégralement close. Puisque le schéma $\operatorname{Spec}(\mathscr{A})$ est normal, il en est de même du $\operatorname{schéma~} \operatorname{Spec}(\mathscr{A}[T])$, ainsi que de son analytifié $\mathbf{A}_{\mathscr{A}}^{1 \text {,an }}$, d'après [Berk93, 2.2.7]. Le domaine affinoïde $\mathscr{M}(\mathscr{A}\{T\})$ de ce dernier est donc, lui aussi, normal, d'après [Berk93, 2.2.1]. Puisque l'anneau $\mathscr{A}\{T\}$ est intègre et normal, il est finalement intégralement clos.

Le lemme suivant met en lumière le lien géométrique recherché.

Lemme 2.2. Quel que soit $x \in \mathbf{D} \backslash\{0\}$, la projection

$$
\mathscr{M}(\mathscr{A}\{T, U\} /(f T-U)) \rightarrow \mathscr{M}(\mathscr{A})=X
$$

induit un isomorphisme

où $\varepsilon=|U(x)|>0$.

$$
\tau^{-1}(x) \stackrel{\sim}{\longrightarrow} V_{\varepsilon} \hat{\otimes}_{k} \mathscr{H}(x)
$$

En particulier, les fibres du morphisme $\tau$ au-dessus des points de $\mathbf{D} \backslash\{0\}$ sont géométriquement réduites. 


\section{ConneXITÉ EN GÉOMÉTRIQUE ANALYTIQUe $p$-ADIQUE}

Démonstration. Soit $x \in \mathbf{D} \backslash\{0\}$. Notons $\mathscr{B}=\mathscr{A} \hat{\otimes}_{k} \mathscr{H}(x)$. L'algèbre de la fibre $\tau^{-1}(x)$ n'est autre que

$$
(\mathscr{A}\{T, U\} /(f T-U)) \hat{\otimes}_{k\{U\}} \mathscr{H}(x) \simeq \mathscr{B}\{T\} /(f T-U(x)) \simeq \mathscr{B}\{\varepsilon T\} /(f T-1),
$$

où $\varepsilon=|U(x)|>0$. On reconnait l'algèbre du domaine affinoïde de $\mathscr{M}(\mathscr{B})$ défini par

$$
\{y \in \mathscr{M}(\mathscr{B})|| f(y) \mid \geqslant \varepsilon\}
$$

et qui est isomorphe à $V_{\varepsilon} \hat{\otimes}_{k} \mathscr{H}(x)$.

Passons à la seconde partie du lemme. L'espace affinoïde $X$ est réduit, donc son domaine affinoïde $V_{\varepsilon}$ l'est aussi, d'après [Berk93, 2.2.1]. Puisque le corps $k$ est algébriquement clos, l'espace $V_{\varepsilon}$ est géométriquement réduit et il en est de même pour $\tau^{-1}(x) \simeq V_{\varepsilon} \hat{\otimes}_{k} \mathscr{H}(x)$, d'après [Duc03a, 4.17].

Pour $\varepsilon \in[0,1]$, notons $\eta_{\varepsilon}$ le point de $\mathbf{D}$ associé à la valeur absolue définie par $\sum_{i \geqslant 0} a_{i} U^{i} \in$ $k\{U\} \mapsto \max _{i \geqslant 0}\left\{\left|a_{i}\right| \varepsilon^{i}\right\} \in \mathbb{R}_{+}$. D'après le lemme, la fibre de $\tau$ au-dessus du point $\eta_{\varepsilon}$ est isomorphe à $V_{\varepsilon} \hat{\otimes} \mathscr{H}\left(\eta_{\varepsilon}\right)$, quel que soit $\left.\left.\varepsilon \in\right] 0,1\right]$.

Afin de pouvoir appliquer les résultats de la section précédent, nous avons besoin d'une propriété de platitude, que nous démontrons ici.

LeMme 2.3. Le morphisme

$$
\tau: \mathscr{M}(\mathscr{A}\{T, U\} /(f T-U)) \rightarrow \mathscr{M}(k\{U\})=\mathbf{D}
$$

est plat.

Démonstration. L'anneau $k\{U\}$ étant principal, il nous suffit de montrer que l'algèbre $\mathscr{A}\{T, U\} /$ $(f T-U)$ ne possède aucun élément de $k\{U\}$-torsion. Il nous suffit même de montrer que le morphisme

$$
t: k\{U\} \rightarrow \mathscr{A}\{T, U\} /(f T-U) \simeq \mathscr{A}\{T\}
$$

est injectif, puisque l'algèbre $\mathscr{A}\{T, U\} /(f T-U) \simeq \mathscr{A}\{T\}$ est intègre. L'interprétation géométrique des fibres du morphisme $\tau$ nous montre que sa fibre au point $\eta_{1}$ n'est pas vide. En particulier, toute fonction $g$ de $k\{U\}$ vérifiant $t(g)=0$ est nulle en $\eta_{1}$ et donc nulle sur $\mathbf{D}$.

Remarque 2.4. La fibre du morphisme $\tau$ au-dessus du point 0 de $\mathbf{D}$ est isomorphe à l'espace $\mathscr{M}(\mathscr{A}\{T\} /(f T))$ et ne saurait donc être réduite lorsque $f$ possède des multiplicités. Ce problème fera l'objet de la prochaine section.

Remarquons néanmoins que si l'hypersurface de $X$ définie par l'équation $f=0$ est réduite, un calcul simple montre que la fibre $\tau^{-1}(0)$ l'est aussi. En outre, elle est connexe, puisqu'elle est réunion de l'espace $X$ et d'une droite au-dessus du lieu d'annulation de $f$ dans $X$. Le théorème 1.10 appliqué au morphisme $\tau$ et au point rigide 0 de $\mathbf{D}$ entraîne alors que le domaine affinoïde $\{x \in X|| f(x) \mid \geqslant \varepsilon\}$ est connexe, dès que $\varepsilon$ est assez petit. S'en déduit, en particulier, l'analogue du théorème de Hartogs.

Intéressons-nous, à présent, à la variation des composantes connexes des domaines $V_{\varepsilon}$, pour $\varepsilon>0$. Énonçons tout d'abord un lemme. Le caractère fini mis à part, nous redémontrons ici, dans un cas élémentaire, le résultat [Berk04, 6.3.1]. Rappelons que nous avons supposé le corps $k$ algébriquement clos et de valuation non triviale. Par conséquent, l'égalité $\sqrt{\left|k^{*}\right|}=\left|k^{*}\right|$ est vérifiée.

Lemme 2.5. La trace d'une partie simple de $\mathbf{D}$ sur le segment

$$
\left\{\eta_{\varepsilon}, 0 \leqslant \varepsilon \leqslant 1\right\} \simeq[0,1]
$$

est une réunion finie d'intervalles dont les bornes sont des éléments de $\left|k^{*}\right| \cup\{0,+\infty\}$.

Démonstration. Puisqu'une partie simple est obtenue, par définition, comme une combinaison booléenne finie de domaines affinoïdes, il suffit de démontrer le résultat pour ces derniers. 


\section{J. Poineau}

D'après le théorème de Gerritzen et Grauert (cf. [Duc03c, 2.4]), tout domaine affinoïde de $\mathbf{D}$ peut s'écrire comme réunion finie de domaines rationnels, eux-mêmes intersections de domaines du type $\{z \in \mathbf{D}|| g(z)|\leqslant| h(z) \mid\}$, où $g$ et $h$ désignent des fonctions analytiques sur $\mathbf{D}$. Remarquons encore que, si $g=\sum_{i \in \mathbb{N}} b_{i} U^{i}$ et $h=\sum_{i \in \mathbb{N}} c_{i} U^{i}$ dans $k\{U\}$, les fonctions, définies de [0,1] dans $\mathbb{R}^{+}$,

$$
\varepsilon \mapsto \max _{i \in \mathbb{N}}\left\{\ln \left(\left|b_{i}\right|\right)+i \ln (\varepsilon)\right\} \quad \text { et } \quad \varepsilon \mapsto \max _{i \in \mathbb{N}}\left\{\ln \left(\left|c_{i}\right|\right)+i \ln (\varepsilon)\right\}
$$

sont linéaires par morceaux. Nous concluons grâce à l'équivalence

$$
\left|g\left(\eta_{\varepsilon}\right)\right| \leqslant\left|h\left(\eta_{\varepsilon}\right)\right| \Longleftrightarrow \max _{i \in \mathbb{N}}\left\{\ln \left(\left|b_{i}\right|\right)+i \ln (\varepsilon)\right\} \leqslant \max _{i \in \mathbb{N}}\left\{\ln \left(\left|c_{i}\right|\right)+i \ln (\varepsilon)\right\}
$$

qui est vérifiée quel que soit $\varepsilon \in[0,1]$.

Venons-en au résultat concernant la variation des composantes connexes. Signalons que si l'on ne s'intéresse qu'à leur nombre, on retrouve un théorème d'Abbes et Saito (cf. [AS02, Theorem 5.1]).

ThÉorème 2.6. Soit $k$ un corps ultramétrique complet algébriquement clos et dont la valuation $n$ 'est pas triviale. Soit $X$ un espace strictement $k$-affinoïde intègre et $f$ une fonction analytique sur $X$ dont la norme spectrale vaut 1 . Soit $m \in] 0,1] \cap \sqrt{\left|k^{*}\right|}$. Alors il existe une partition finie $\mathcal{P}$ de $[m, 1]$ en intervalles vérifiant la condition suivante: quel que soit $I \in \mathcal{P}$, quels que soient $\varepsilon^{\prime}, \varepsilon \in I$, avec $\varepsilon^{\prime} \leqslant \varepsilon$, l'inclusion

$$
\{x \in X|| f(x) \mid \geqslant \varepsilon\} \subset\left\{x \in X|| f(x) \mid \geqslant \varepsilon^{\prime}\right\}
$$

induit une bijection

$$
\pi_{0}(\{x \in X|| f(x) \mid \geqslant \varepsilon\}) \rightarrow \pi_{0}\left(\left\{x \in X|| f(x) \mid \geqslant \varepsilon^{\prime}\right\}\right) .
$$

En outre, les bornes des intervalles sont des éléments de $\left|k^{*}\right| \cup\{0,+\infty\}$.

Démonstration. Notons $V$ le domaine strictement $k$-affinoïde de $\mathbf{D}$ défini par

$$
V=\{z \in \mathbf{D}|| U(z) \mid \geqslant m\} .
$$

D'après le lemme 1.1, le morphisme $\tau^{\prime}$ déduit de $\tau$ par le changement de base $V \hookrightarrow \mathbf{D}$ est plat. Ses fibres, isomorphes, après extension du corps de base, à des espaces du type $V_{\varepsilon}$, avec $\varepsilon>0$, sont géométriquement réduites et déployées. D'après le théorème 1.9 , il existe donc une partition finie $\mathcal{P}$ de $V$ en parties simples de domaines affinoïdes au-dessus desquelles le morphisme $\tau^{\prime}$ admet un découpage. Considérons l'un des morphismes quasi-étales $\varphi: Z \rightarrow V$, où $Z$ est un espace $k$-analytique compact, intervenant dans le découpage.

Le raisonnement qui suit fait intervenir, dans un cas simple, la notion de squelette. On la trouvera introduite dans [Berk99, §5]. L'espace $k$-affinoïde $V$ est isomorphe à la fibre générique du $k^{\circ}$-schéma formel pluristable non dégénéré

$$
\mathscr{V}=\operatorname{Spf}\left(k^{\circ}\{U, V\} /(U V-\alpha)\right),
$$

où $k^{\circ}$ désigne l'anneau de valuation de $k$ et $\alpha$ un élément de $k^{\circ}$ de valeur absolue $m$. Le squelette $\mathrm{S}(\mathscr{V})$ du schéma formel $\mathscr{V}$ est le segment

$$
J=\left\{\eta_{\varepsilon}, m \leqslant \varepsilon \leqslant 1\right\} \simeq[m, 1],
$$

tracé sur la fibre générique $\mathscr{V}_{\eta} \simeq V$. D'après [Duc03b, 3.1], il existe alors une unique structure $\sqrt{\left|k^{*}\right|}$-linéaire par morceaux (au sens de $\left[\operatorname{Berk04,\S 1])~sur~} \Delta=\varphi^{-1}(\mathrm{~S}(\mathscr{V})\right.$ ) telle que l'application

$$
\varphi_{\mid \Delta}: \Delta \rightarrow \mathrm{S}(\mathscr{V})
$$

soit linéaire par morceaux et soit G-localement une immersion. En particulier, puisque $\Delta$ est compact, il existe une partition finie de $\Delta$ en parties linéaires qui sont homéomorphes à leur image 


\section{ConneXITÉ EN GÉOMÉTRIQUE ANALYTIQUe $p$-ADIQUE}

par $\varphi_{\mid \Delta}$, elle-même linéaire. Pour chaque image $Q$, nous pouvons construire, à partir de la section associée à $\varphi$, une section de $\tau^{\prime}$ au-dessus de $Q$.

En procédant de même pour chaque morphisme étale, nous obtenons finalement, pour chaque élément $P$ de $\mathcal{P}$, une partition $\mathcal{Q}_{P}$ de $P \cap J$ en un nombre fini de parties linéaires et, au-dessus de chaque $Q \in \mathcal{Q}_{P}$, un ensemble fini $\mathcal{T}$ de sections de $\tau^{\prime}$ au-dessus de $Q$ vérifiant la condition suivante: quel que soit $z \in Q$, chaque composante connexe de la fibre $\tau^{\prime-1}(z)$ contient un et un seul élément de la forme $t(z)$, avec $t \in \mathcal{T}$.

Examinons, à présent, la forme des parties $Q$ considérées précédemment. Nous souhaitons montrer qu'elles sont réunions finies d'intervalles à coordonnées dans $\sqrt{\left|k^{*}\right|} \cup\{0,+\infty\}$. C'est le cas pour les traces des éléments de $\mathcal{P}$ sur $J$, d'après le lemme 2.5 , et donc pour leurs parties $\sqrt{\left|k^{*}\right|}$-linéaires par morceaux.

Finalement, les sections de $\tau^{\prime}$ définies précédemment sont définies sur des intervalles contenus dans $J$. Soient $I$ un tel intervalle et $\varepsilon \in I$. Rappelons que l'image de la fibre de $\tau^{\prime}$ au-dessus de $\eta_{\varepsilon}$ par le morphisme

$$
\pi: \mathscr{M}(\mathscr{A}\{T, U\} /(f T-U)) \rightarrow \mathscr{M}(\mathscr{A})=X
$$

est isomorphe au domaine affinoïde $V_{\varepsilon}$. Pour $\varepsilon^{\prime} \in I, \varepsilon^{\prime} \leqslant \varepsilon$, les images des sections par le morphisme $\pi$ joignent les composantes connexes de $V_{\varepsilon}$ à celles de $V_{\varepsilon}^{\prime}$. Puisque les différentes sections aboutissent à des composantes connexes distinctes et que toutes sont atteintes, on en déduit que les composantes connexes de $V_{\varepsilon}$ sont les traces de celles de $V_{\varepsilon}^{\prime}$.

\section{3. Élimination des multiplicités}

Conservons les hypothèses de la partie précédente: le corps $k$ est un corps algébriquement clos dont la valuation n'est pas triviale, l'espace $X$ un espace strictement $k$-affinoïde intègre et la norme spectrale de la fonction $f$ vaut 1 . Nous démontrons ici le théorème 1 dans ce cas particulier.

Remarquons que, puisque le morphisme de normalisation est continu et surjectif, nous pouvons, quitte à remplacer $X$ par son normalisé, supposer que l'espace $X$ est normal. D'après [Duc03a, 4.18], les domaines $V_{\varepsilon}$, pour $\varepsilon>0$, sont alors normaux. Il nous suffit donc de montrer qu'ils sont connexes, pour $\varepsilon$ assez petit.

D'après la section précédente, nous disposons d'un morphisme plat,

$$
\tau: \mathscr{M}(\mathscr{A}\{T, U\} /(f T-U)) \rightarrow \mathbf{D},
$$

dont seule la fibre au-dessus du point 0 peut présenter des multiplicités. Nous allons montrer qu'il est possible de modifier ce morphisme de façon que toutes ses fibres deviennent réduites.

Dans les raisonnements qui suivent, nous quittons le domaine des espaces analytiques pour celui des schémas. Il nous faut donc introduire de nouveaux objets. Soient $D=\operatorname{Spec}(k\{U\}), F=$ $\operatorname{Spec}(\mathscr{A}\{T, U\} /(f T-U))$ et $\alpha: F \rightarrow D$ le morphisme induit par $t: k\{U\} \rightarrow \mathscr{A}\{T, U\} /(f T-U)$. Soient $x$ un point fermé de $D \backslash\{0\}$ et $\mathbf{x}$ le point rigide de $\mathbf{D}$ qui lui correspond. La fibre de $\alpha$ au-dessus de $x$ a même anneau que la fibre de $\tau$ au-dessus de $\mathbf{x}$. En particulier, elle est réduite.

D'après le lemme 2.1, le schéma $F$ est normal. Le problème de réduction des fibres auquel nous sommes confrontés se ramène donc à un problème de multiplicités génériques. En effet, une hypersurface principale d'un schéma normal et noethérien est réduite si, et seulement si, elle est génériquement réduite (c'est une conséquence de la condition $\left(\mathrm{S}_{2}\right)$, cf. [Mat80, 17.I]).

Rappelons que si $R$ et $S$ sont deux anneaux de valuation discrète et que $S$ domine $R$, on dit que $S$ est faiblement non ramifié au-dessus de $R$ lorsque l'idéal maximal de $R$ engendre l'idéal maximal de $S$. Si $x$ désigne un point fermé de $D$, la remarque précédente entraîne que la fibre $\alpha^{-1}(x)$ au-dessus de $x$ est réduite si, et seulement si, l'anneau $\mathscr{O}_{F, \eta}$ est faiblement non ramifié au-dessus de l'anneau 


\section{J. POINEAU}

$\mathscr{O}_{D, x}$, pour tout point générique $\eta$ de $\alpha^{-1}(x)$. Aussi les méthodes que nous mettrons en œuvre viseront-elles à éliminer la ramification. Dans le cas où elle est modérée, le lemme d'Abhyankar nous montre qu'il est possible d'y parvenir, après un nombre fini d'extensions de Kummer sur la base. Pour traiter le cas général, nous utiliserons le théorème que démontre Epp dans [Epp73]. Rappelons-en l'énoncé, sous la forme corrigée qu'en proposent Oesterlé et Pharamond dit d'Costa [OP97, appendice, théorème 2] :

ThÉorème 3.1 (Epp). Soient $A$ et $A^{\prime}$ deux anneaux de valuation discrète, $K$ et $K^{\prime}$ leur corps de fractions, $k$ et $k^{\prime}$ leur corps résiduel. On suppose que $A^{\prime}$ domine $A$. Si la caractéristique $p$ de $k$ n'est pas nulle, on suppose que les éléments de $k^{\prime p^{\infty}}$, le plus grand sous-corps parfait de $k^{\prime}$, sont algébriques et séparables sur $k$. Il existe alors une extension algébrique $K_{1}$ de degré fini de $K$ telle que:

(a) la fermeture intégrale $A_{1}$ de $A$ dans $K_{1}$ soit un $A$-module de type fini et un anneau de valuation discrète;

(b) si $K_{1}^{\prime}$ est une extension composée de $K_{1}$ et $K^{\prime}$, tout anneau de valuation discrète $A_{1}^{\prime}$ de corps des fractions $K_{1}^{\prime}$ qui domine $A^{\prime}$ est faiblement non ramifié au-dessus de $A_{1}$.

Nous aurons besoin d'utiliser le fait que la propriété d'être faiblement non ramifié reste stable par certaines opérations. Le résultat suivant se déduit sans peine de la proposition 1 de l'appendice du même article [OP97].

Proposition 3.2. Soient $A$ et $A^{\prime}$ deux anneaux de valuation discrète, $K$ et $K^{\prime}$ leur corps de fractions, $k$ et $k^{\prime}$ leur corps résiduel. On suppose que $A^{\prime}$ domine $A$ et que $A^{\prime}$ est faiblement non ramifié au-dessus de $A$. Soit $A_{1}$ un anneau de valuation discrète dont le corps des fractions $K_{1}$ est une extension algébrique de degré fini de $K$ et dont le corps résiduel $k_{1}$ est une extension séparable de $k$. Alors, si $K_{1}^{\prime}$ est une extension composée de $K_{1}$ et $K^{\prime}$, tout anneau de valuation discrète $A_{1}^{\prime}$ de corps des fractions $K_{1}^{\prime}$ qui domine $A^{\prime}$ est faiblement non ramifié au-dessus de $A_{1}$.

Notons $\eta_{1}, \ldots, \eta_{p}$, avec $p \in \mathbb{N}^{*}$, les points génériques de la fibre du morphisme $\alpha$ au-dessus de 0 . Pour chaque $i \in \llbracket 1, p \rrbracket$, nous allons appliquer le théorème de Epp aux anneaux de valuation discrète $\mathscr{O}_{D, 0}$ et $\mathscr{O}_{F, \eta_{i}}$. Les hypothèses en sont vérifiées, en vertu du résultat suivant.

Lemme 3.3. Supposons que la caractéristique du corps algébriquement clos $k$ ne soit pas nulle. Soit $\mathscr{B}$ une algèbre strictement $k$-affinoïde intègre. Alors le plus grand sous-corps parfait contenu dans le corps des fractions de $\mathscr{B}$ est égal à $k$.

Démonstration. D'après le lemme de normalisation de Noether [BGR84, 6.1.2/2], il existe $d \in \mathbb{N}$ et un morphisme fini $\varphi: k\left\{T_{1}, \ldots, T_{d}\right\} \rightarrow \mathscr{B}$. La conclusion du lemme est vérifiée pour le corps $\operatorname{Frac}\left(k\left\{T_{1}, \ldots, T_{d}\right\}\right)$, car l'anneau $k\left\{T_{1}, \ldots, T_{d}\right\}$ est factoriel. D'après [Epp73, $\left.\S 0.4\right]$, elle l'est encore après toute extension finie, ce qui s'applique, en particulier, à $\operatorname{Frac}(\mathscr{B})$.

Quel que soit $i \in \llbracket 1, p \rrbracket$, nous obtenons ainsi une extension algébrique finie $K_{\eta_{i}}$ de $\operatorname{Frac}(k\{U\})$ vérifiant les conclusions du théorème de Epp.

Soient $K_{1}$ une extension finie de $k\{U\}$ dans laquelle s'injectent tous les corps $K_{\eta_{i}}$, avec $i \in \llbracket 1, p \rrbracket$, et engendrée par les images de ces corps. Notons $\mathscr{N}$ la fermeture intégrale de $k\{U\}$ dans $K_{1}$. Nous allons, à présent, considérer le spectre de la fermeture intégrale de $\mathscr{A}\{T, U\} /(f T-U)$ dans un composé du corps de ses fractions et de $K_{1}$. Géométriquement, cela revient à considérer le produit fibré de $F$ par $\Delta=\operatorname{Spec}(\mathscr{N})$ au-dessus de $D$, puis à le normaliser. Notons $G=\operatorname{Spec}(\mathscr{G})$ le schéma 


\section{ConneXITÉ EN GÉOMÉTRIQUE ANALYTIQUe $p$-ADIQUE}

ainsi obtenu.

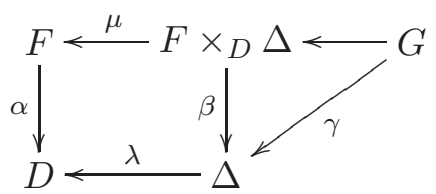

Commençons par énoncer quelques remarques sur les morphismes et les espaces apparaissant dans le diagramme.

(a) Le morphisme surjectif $\lambda: \Delta=\operatorname{Spec}(\mathscr{N}) \rightarrow D$ est plat et surjectif. Puisque l'anneau $k\{U\}$ est excellent, il est également fini. En particulier, l'anneau $\mathscr{N}$ est un anneau de Dedekind et une algèbre strictement $k$-affinoïde.

(b) Le morphisme $\mu: F \times{ }_{D} \Delta \rightarrow F$ est, lui aussi, fini, plat et surjectif. Le morphisme $G \rightarrow F$ est donc encore fini et l'anneau $\mathscr{G}$ est une algèbre strictement $k$-affinoïde. On en déduit également que toutes les composantes connexes de $G$ se surjectent sur $F$.

(c) Le morphisme $\beta: F \times{ }_{D} \Delta \rightarrow \Delta$ est plat et surjectif.

(d) Le morphisme $\gamma$ est surjectif. Il est également plat, puisque, quelle que soit la composante connexe $H$ de $G$, l'anneau de Dedekind $\mathscr{N}$ s'injecte dans l'anneau de $H$, qui est intègre et donc sans torsion.

Établissons encore deux propriétés, moins immédiates :

Lemme 3.4. Le schéma $F \times_{D} \Delta$ est normal hors des fibres de $\alpha \circ \mu$ au-dessus du point 0 . En particulier, si $x$ est un point de $\Delta \backslash \lambda^{-1}(0)$, alors la fibre de $\gamma$ au-dessus de $x$ est isomorphe à celle de $\beta$ au-dessus de $x$ et donc à celle de $\alpha$ au-dessus de $\lambda(x)$.

Démonstration. Remarquons, tout d'abord, que les fibres du morphisme $\alpha$ autres que la fibre audessus de 0 sont toutes normales. En effet, pour la fibre générique, c'est évident et cela découle de l'interprétation géométrique des fibres du morphisme $\tau$ pour les points fermés. Puisqu'en outre, $\alpha$ est plat, le morphisme $F \backslash \alpha^{-1}(0) \rightarrow D \backslash\{0\}$ est normal, au sens de [Gro65, 6.8.1]. Puisque $\Delta \backslash \lambda^{-1}(0)$ est un schéma normal, on en déduit que $\left(F \backslash \alpha^{-1}(0)\right) \times_{D \backslash\{0\}}\left(\Delta \backslash \lambda^{-1}(0)\right)$ est encore normal, en vertu de [Gro65, 6.14.1].

Lemme 3.5. Les fibres du morphisme $\gamma$ sont géométriquement réduites et déployées.

Démonstration. Les fibres du morphisme $\alpha$, à l'exception éventuelle de $\alpha^{-1}(0)$, sont géométriquement réduites et déployées. On en déduit, à l'aide du lemme précédent, que les fibres du morphisme $\gamma$ au-dessus des points de $\Delta \backslash \lambda^{-1}(0)$ le sont encore. Il nous reste à considérer les fibres au-dessus de $\lambda^{-1}(0)$, qui est une réunion finie de points fermés. Puisque le corps de base $k$ est supposé algébriquement clos, elles sont évidemment encore déployées.

Soient $x$ un point fermé de $\Delta$ et $H$ une composante connexe de $G$. La fibre du morphisme $\gamma_{H}$, induit par $\gamma$ sur $H$, au-dessus de ce point est une hypersurface principale différente de $H$. Soit $\zeta$ l'un de ses points génériques. Il est de codimension 1 dans $H$, tout comme l'est son image $\eta$ dans $F$, par les théorèmes de Cohen et Seidenberg. Le point $\eta$ est un donc un point générique de la fibre de $\alpha$ au-dessus du point fermé $\lambda(x)$ de $D$ et l'anneau local $\mathscr{O}_{H, \zeta}$ est un anneau de valuation discrète dominant $\mathscr{O}_{F, \eta}$ et dont le corps des fractions coïncide avec le corps des fonctions de $H$. La construction de $G$ et la proposition 3.2 nous permettent alors d'affirmer que l'anneau de valuation discrète $\mathscr{O}_{G, \zeta}=\mathscr{O}_{H, \zeta}$ est faiblement non ramifié au-dessus de $\mathscr{O}_{\Delta, x}$. Par conséquent, la fibre $\gamma^{-1}(x)$ est génériquement réduite, et donc réduite, puisqu'il s'agit d'une hypersurface principale d'un schéma normal et noethérien. 


\section{J. POINEAU}

Revenons, à présent, à des morphismes entre espaces analytiques.

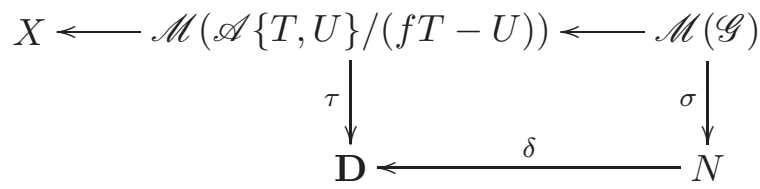

Soit $\omega$ un point rigide de $N=\mathscr{M}(\mathscr{N})$ qui s'envoie sur $0 \in \mathbf{D}$ par le morphisme $\delta: N \rightarrow \mathbf{D}$. D'après le lemme 3.4, il existe un voisinage affinoïde $V$ de $\omega$ dans $N$ tel que, pour tout point rigide $v$ de $V \backslash\{\omega\}$, la fibre du morphisme $\sigma: \mathscr{M}(\mathscr{G}) \rightarrow N$ au-dessus de $v$ soit isomorphe à $V_{\varepsilon} \hat{\otimes}_{k} \mathscr{H}(w)$, où $w=\delta(v), \varepsilon=|U(w)|>0$ et $V_{\varepsilon}=\{x \in X|| f(x) \mid \geqslant \varepsilon\}$.

Toutes les conditions sont, à présent, réunies pour que nous puissions appliquer le théorème 1.9 au morphisme $\sigma$ au voisinage du point $\omega$. En effet, le morphisme $\sigma$ est plat et à fibres géométriquement réduites et déployées, car $\gamma$ l'est. Le théorème nous assure l'existence d'une partie simple $P$ d'un domaine affinoïde de $V$, de morphisme quasi-étales et de sections satisfaisant certaines conditions. Rappelons qu'une partie simple contenant un point rigide contient toujours un voisinage de ce point. Considérons un morphisme quasi-étale $e: U \rightarrow V$ dont l'image contient $P$. Choisissons un point rigide $\omega^{\prime}$ de $U$ qui soit un antécédent de $\omega$ par $e$. Puisque le corps de base $k$ est algébriquement clos, le corps résiduel complété $\mathscr{H}(\omega)$ l'est également et le morphisme $e$ induit un isomorphisme

$$
\mathscr{H}(\omega) \stackrel{\sim}{\longrightarrow} \mathscr{H}\left(\omega^{\prime}\right) .
$$

Puisque les points $\omega$ et $\omega^{\prime}$ sont rigides et donc intérieurs, on en déduit qu'il existe un isomorphisme local entre un voisinage affinoïde de $\omega^{\prime}$ et un voisinage affinoïde de $\omega$, en vertu de [Berk93, 3.4.1].

En composant les différents isomorphismes réciproques par les sections du théorème et en restreignant de façon adéquate, nous déduisons finalement l'existence d'un voisinage $V^{\prime}$ de $\omega$ dans $V$ et d'une famille finie $\mathcal{T}$ de sections de $\sigma$ sur $V^{\prime}$ satisfaisant la condition suivante: pour tout point $v$ de $V^{\prime}$, chaque composante connexe de la fibre du morphisme $\sigma$ au-dessus de $v$ contient un et un seul élément de la forme $t(v)$, avec $t \in \mathcal{T}$. Remarquons que nous pouvons supposer que $V^{\prime}$ est connexe par arcs, puisque $N$ l'est localement.

Puisque le morphisme $\delta: N \rightarrow \mathbf{D}$ est fini et plat, il est ouvert au voisinage des points rigides de $N$, donc il existe $\varepsilon>0$ tel que l'image de $V^{\prime}$ par $\delta$ contienne l'ensemble $\{d \in \mathbf{D}|| U(d) \mid \leqslant \varepsilon\}$. Soit $v$ un élément de $V^{\prime}$ tel que $|U(\delta(v))|=\varepsilon$. Soit $\left.\left.\varepsilon^{\prime} \in\right] 0, \varepsilon\right]$. Il existe un chemin continu $l$ dans $V^{\prime}$ joignant $v$ à un point $v^{\prime}$ vérifiant les deux conditions

$$
|U(\delta(l))| \subset\left[\varepsilon^{\prime}, \varepsilon\right] \text { et }\left|U\left(\delta\left(v^{\prime}\right)\right)\right|=\varepsilon^{\prime} .
$$

Pour chaque $t \in \mathcal{T}$, l'image du chemin $l$ par la section $t$ fournit un chemin $l_{t}$ dans $\mathscr{M}(\mathscr{G})$.

Projetons, à présent, les chemins ainsi construits dans $X$ par le morphisme

$$
\mathscr{M}(\mathscr{G}) \rightarrow \mathscr{M}(\mathscr{A}\{T, U\} /(f T-U)) \rightarrow X=\mathscr{M}(\mathscr{A}) .
$$

Quel que soit $w \in l$, chaque composante connexe de $\sigma^{-1}(w)$ coupe un et un seul des chemins $l_{t}$, avec $t \in \mathcal{T}$. Les images de ces chemins joignent donc les composantes connexes de $V_{\varepsilon}$ aux composantes connexes de $V_{\varepsilon^{\prime}}$. En particulier, les composantes connexes de $V_{\varepsilon}$ sont les traces de celles de $V_{\varepsilon^{\prime}}$ et donc les traces de celles de $\{x \in X / f(x) \neq 0\}$. Or, d'après [Bar70] ou [Lut74], le complémentaire de l'hypersurface définie par $f$ dans $X$ est connexe, dès que $X$ est connexe. Par conséquent, le domaine affinoïde $V_{\varepsilon}$ est connexe. Nous avons finalement démontré le résultat suivant.

THÉORÈme 3.6. Soient $k$ un corps ultramétrique complet algébriquement clos dont la valuation $n$ 'est pas triviale, $X$ un espace strictement $k$-affinoïde intègre et $f$ une fonction analytique sur $X$ 


\section{ConneXITÉ EN GÉOMÉTRIQUE ANALYTIQUe $p$-ADIQUE}

dont la norme spectrale vaut 1 . Alors le domaine affinoïde de $X$ défini par

$$
\{x \in X|| f(x) \mid \geqslant \varepsilon\}
$$

est irréductible, dès que $\varepsilon$ est assez petit.

\section{Démonstration des théorèmes annoncés}

Dans cette section, nous expliquons comment déduire les théorèmes 1 et 2 en toute généralité à partir de ceux démontrés dans les deux sections précédentes. Fixons $k$ un corps ultramétrique complet et $X$ un espace $k$-affinoïde d'algèbre $\mathscr{A}$.

Intéressons-nous, tout d'abord, au théorème 1 . Soient $n \in \mathbb{N}^{*}$ et $f_{1}, \ldots, f_{n}$ des fonctions analytiques sur $X$. Quel que soit $\varepsilon=\left(\varepsilon_{1}, \ldots, \varepsilon_{n}\right) \in\left(\mathbb{R}_{+}^{*}\right)^{n}$, nous noterons $V_{\boldsymbol{\varepsilon}}$ le domaine analytique de $X$ défini par

$$
V_{\varepsilon}=\bigcup_{1 \leqslant j \leqslant n}\left\{x \in X|| f_{j}(x) \mid \geqslant \varepsilon_{j}\right\} .
$$

Supposons que l'espace $X$ soit irréductible et montrons que le domaine affinoïde $V_{\varepsilon}$ est irréductible, dès que $\varepsilon$ est assez petit. Comme dans la section précédente, puisque le morphisme de normalisation est continu et surjectif, nous pouvons, quitte à remplacer $X$ par son normalisé, supposer que l'espace $X$ est normal.

Soit $j \in \llbracket 1, n \rrbracket$. Commençons par nous intéresser aux espaces affinoïdes du type

$$
V_{j, \varepsilon}=\left\{x \in X|| f_{j}(x) \mid \geqslant \varepsilon\right\},
$$

avec $\varepsilon>0$. Soit $K$ un corps ultramétrique complet algébriquement clos contenant $k$ tel que l'espace $X \hat{\otimes} K$ soit strictement $K$-affinoïde. Le résultat que nous cherchons à démontrer est évident lorsque la fonction $f_{j}$ est nulle. Nous excluons dorénavant ce cas. D'après [BGR84, 6.2.1/4 (ii)], il existe alors $c \in K^{*}$ et $m \in \mathbb{N}^{*}$ tels que $\left|c f_{j}^{m}\right|_{\text {sup }}=1$. Nous pouvons donc supposer que la norme spectrale de $f_{j}$ vaut 1 , quitte à remplacer $f_{j}$ par $c f_{j}^{m}$, les domaines affinoïdes en jeu étant alors liés par la relation

$$
\left\{x \in X \hat{\otimes}_{k} K|| c f_{j}^{m}(x) \mid \geqslant \varepsilon\right\}=\left\{x \in X \hat{\otimes}_{k} K|| f_{j}(x) \mid \geqslant\left(\frac{\varepsilon}{|c|}\right)^{1 / m}\right\} .
$$

L'espace strictement $K$-affinoïde $X \hat{\otimes}_{k} K$ possède un nombre fini $Z_{1}, \ldots, Z_{r}$, avec $r \in \mathbb{N}$, de composantes irréductibles. Sur chacune d'elles, le théorème 1 est valable, d'après le théorème 3.6. Par conséquent, il existe $\varepsilon^{\prime}>0$ tel que, quel que soit $i \in \llbracket 1, r \rrbracket$ et quel que soit $\left.\left.\varepsilon \in\right] 0, \varepsilon^{\prime}\right]$, l'espace

$$
\left\{z \in Z_{i}|| f_{j}(z) \mid \geqslant \varepsilon\right\}
$$

soit connexe.

Pour $i \in \llbracket 1, r \rrbracket$, notons $Y_{i}$ l'image de $Z_{i}$ dans $X$. Quitte à imposer un nouvel ordre sur les indices, nous pouvons supposer qu'il existe $s \in \llbracket 1, r \rrbracket$ tel que $Z_{1}, \ldots, Z_{s}$ ne soient pas contenus dans

$$
V_{+}=\left\{x \in X \mid f_{j}(x) \neq 0\right\}
$$

et que $Z_{s+1}, \ldots, Z_{r}$ le soient. Pour $i \in \llbracket 1, s \rrbracket$, choisissons un point $P_{i}$ de $Y_{i}$ en lequel $f_{j}$ ne s'annule pas. D'après [Bar70] ou [Lut74], l'espace $V_{+}$est connexe et même connexe par arcs, en vertu de [Berk90, 3.2.1]. Par conséquent, quel que soit $i \in \llbracket 2, s \rrbracket$, il existe un chemin joignant $P_{1}$ à $P_{i}$ sur lequel $f_{j}$ ne s'annule jamais. Ce chemin étant compact, la fonction $f_{j}$ y atteint son minimum $\varepsilon_{i}^{\prime}>0$.

Posons $\varepsilon_{j}=\min \left(\varepsilon^{\prime}, \varepsilon_{2}^{\prime}, \ldots, \varepsilon_{s}^{\prime}\right)$. Soit $\left.\varepsilon \in\right] 0, \varepsilon_{j}[$. Puisque le morphisme de changement de base

$$
X \hat{\otimes}_{k} K \rightarrow X
$$




\section{J. Poineau}

est continu et surjectif, le domaine affinoïde $V_{j, \varepsilon}$ est connexe. D'après [Duc03a, 4.18], il est également normal, car $X$ est normal. On en déduit qu'il est donc irréductible.

Nous pouvons, sans perte de généralité, supposer qu'aucune des fonctions $f_{j}$, avec $j \in \llbracket 1, n \rrbracket$, n'est nulle. Puisque $X$ est irréductible, il existe un point $x$ de $X$ en lequel aucune des fonctions $f_{j}$, avec $j \in \llbracket 1, n \rrbracket$, ne s'annule. Soit $\left.\varepsilon \in \prod_{j=1}^{n}\right] 0, \min \left(\varepsilon_{j},\left|f_{j}(x)\right|\right)\left[\right.$. Le domaine analytique $V_{\boldsymbol{\varepsilon}}$ est alors réunion de parties connexes dont l'intersection contient un voisinage de $x$ dans $X$. Le lemme suivant nous montre qu'il est irréductible.

Lemme 4.1. Soient $V$ et $W$ deux domaines analytiques irréductibles de $X$. Si l'intérieur de l'intersection $V \cap W$ n'est pas vide, alors la réunion $V \cup W$ est irréductible.

Démonstration. Supposons que le domaine analytique $V \cup W$ soit connexe. Soient $Y$ et $Z$ deux fermés de Zariski de $V \cup W$ dont la réunion recouvre $V \cup W$. Supposons que $Y \neq V \cup W$. Nous avons alors $Y \cap V \neq V$ ou $Y \cap W \neq W$. Nous pouvons supposer que $Y \cap V \neq V$. Par irréductibilité de $V$, nous avons alors $Z \cap V=V$, autrement dit, $V \subset Z$. Par irréductibilité de $W$, nous devons avoir $W \subset Y$ ou $W \subset Z$.

Supposons, par l'absurde que l'on ait $W \subset Y$. Nous avons alors $V \cap W \subset Y \cap Z$. Le domaine analytique d'intérieur non vide $V \cap W$ de $V$ est donc contenu dans le fermé de Zariski non trivial $Y \cap Z$ du domaine analytique irréductible $V$. D'après [Berk90, 3.3.21], cette situation est impossible.

Finalement, nous avons $W \subset Z$ et donc $V \cup W \subset Z$. Par conséquent, le domaine analytique $V \cup W$ est irréductible.

Passons à la démonstration du théorème 2. Soit $f$ une fonction analytique sur $X$. Nous noterons $R_{X}$ le sous-Q-espace vectoriel de $\mathbb{R}_{+}^{*}$ engendré par les valeurs non nulles de la norme spectrale sur l'algèbre $k$-affinoïde $\mathscr{A}$. En particulier, si $X$ est strictement $k$-affinoïde, on a $R_{X}=\sqrt{\left|k^{*}\right|}$. Cette définition est justifiée par le lemme suivant.

LEMme 4.2. Il existe un corps $L$ ultramétrique complet algébriquement clos et de valuation non triviale contenant $k$ tel que l'espace $X \hat{\otimes}_{k} L$ soit strictement $L$-affinoïde. Un tel corps peut être choisi de façon à vérifier en outre

$$
\left|L^{*}\right|=\sqrt{\left|L^{*}\right|}=R_{X}
$$

Dans un premier temps, nous nous intéresserons à la partie du théorème 2 concernant les composantes connexes. L'espace $X$ n'est plus supposé irréductible. Nous utiliserons la définition suivante.

DÉfinition 4.3. Soient $Y$ un espace $k$-analytique et $g$ une fonction analytique sur $Y$. Nous dirons qu'un intervalle $I$ de $\mathbb{R}^{+}$est régulier pour la fonction $g$ sur l'espace $Y$ si, quels que soient $\varepsilon^{\prime}, \varepsilon \in I$, avec $\varepsilon^{\prime} \leqslant \varepsilon$, l'application

$$
\pi_{0}(\{y \in Y|| g(y) \mid \geqslant \varepsilon\}) \rightarrow \pi_{0}\left(\left\{y \in Y|| g(y) \mid \geqslant \varepsilon^{\prime}\right\}\right)
$$

induite par l'inclusion est bijective.

D'après [Duc03a, 5.5], le nombre de composantes connexes géométriques de $X$ et de ses domaines affinoïdes reste inchangé lorsque l'on étend le corps de base. Par conséquent, quitte à changer $k$ en le corps $L \mathrm{du}$ lemme précédent, nous pouvons supposer que le corps $k$ est algébriquement clos, de valuation non triviale et que l'espace $X$ est strictement $k$-affinoïde. Il nous faudra cependant remplacer $\sqrt{\left|k^{*}\right|}$ par $\sqrt{\left|L^{*}\right|}=\left|L^{*}\right|=R_{X}$.

Afin de réduire encore notre problème, nous aurons besoin du lemme suivant.

Lemme 4.4. Supposons que l'espace $X$ soit réunion de deux fermés de Zariski $Y$ et $Z$ sur lesquels il existe une partition finie de $\mathbb{R}^{+}$en intervalles réguliers pour $f$ et dont les bornes sont des éléments de $\sqrt{\left|k^{*}\right|} \cup\{0,+\infty\}$. Alors, la même propriété vaut sur $X$. 
Démonstration. Quel que soit $\varepsilon>0$, nous noterons

$$
V_{\varepsilon}^{\prime}=\{x \in Y|| f(x) \mid \geqslant \varepsilon\} \quad \text { et } \quad V_{\varepsilon}^{\prime \prime}=\{x \in Z|| f(x) \mid \geqslant \varepsilon\} .
$$

Soit $I$ un intervalle de $\mathbb{R}^{+}$qui soit régulier pour $f$ à la fois sur $Y$ et sur $Z$ et dont les bornes sont des éléments de $\sqrt{\left|k^{*}\right|} \cup\{0,+\infty\}$. Il suffit de montrer qu'un tel intervalle admet une partition finie en intervalles réguliers pour $f$ sur $X$ avec la même condition sur les bornes. Nous pouvons supposer que, quel que soit $\varepsilon \in I$, l'espace affinoïde $V_{\varepsilon}$ n'est pas vide.

Soit $\alpha \in I$. Notons $C_{1}, \ldots, C_{r}$, avec $r \in \mathbb{N}$, les composantes connexes de $V_{\alpha}^{\prime}$ et $C_{r+1}, \ldots, C_{s}$, avec $s \in \mathbb{N}$, celles de $V_{\alpha}^{\prime \prime}$. Pour $\varepsilon \in I$ et $i \in \llbracket 1, r \rrbracket$, nous noterons $C_{i, \varepsilon}$ l'unique composante connexe de $V_{\varepsilon}^{\prime}$ qui vérifie

$$
C_{i, \varepsilon} \cap V_{\max (\varepsilon, \alpha)}^{\prime}=C_{i} \cap V_{\max (\varepsilon, \alpha)}^{\prime} .
$$

Pour $\varepsilon \in I$ et $j \in \llbracket r+1, s \rrbracket$, on définit de même une composante connexe $C_{j, \varepsilon}$ de $V_{\varepsilon}^{\prime \prime}$.

Soit $\varepsilon \in I$. Remarquons que toute composante connexe $C$ de $V_{\varepsilon}$ s'écrit de manière unique sous la forme

$$
C=\bigcup_{i \in P} C_{i, \varepsilon},
$$

où $P$ désigne une partie de $\llbracket 1, s \rrbracket$. Définissons l'application

$$
c_{\varepsilon}: \llbracket 1, s \rrbracket \rightarrow \mathcal{P}(\llbracket 1, s \rrbracket)
$$

qui à un entier $i \in \llbracket 1, s \rrbracket$ associe l'ensemble des entiers $j \in \llbracket 1, s \rrbracket$ tels que $C_{j, \varepsilon}$ et $C_{i, \varepsilon}$ soient contenus dans la même composante connexe de $V_{\varepsilon}$.

L'application $c: \varepsilon \mapsto c_{\varepsilon}$ ne peut prendre qu'un nombre fini de valeurs et est décroissante, au sens où, pour $\varepsilon^{\prime} \geqslant \varepsilon$, on a

$$
\forall i \in \llbracket 1, s \rrbracket, \quad c_{\varepsilon^{\prime}}(i) \subset c_{\varepsilon}(i) .
$$

Par conséquent, il existe une partition finie $\mathcal{P}$ de $I$ en intervalles sur lesquels l'application $c$ est constante. Chacun de ces intervalles est régulier pour $f$ sur $X$.

Soit $\beta \in \sqrt{\left|k^{*}\right|}$ tel que l'application $c$ soit constante sur l'intervalle $I \cap[0, \beta]$. Les parties $C_{1, \beta}, \ldots, C_{s, \beta}$ sont alors des domaines strictement affinodes de $V_{\beta}$ et donc de $X$. Nous pouvons choisir les intervalles de la partition $\mathcal{P}$ de façon que leurs bornes différentes de celles de l'intervalle $I$ soient contenues dans l'ensemble $E$ des éléments $\varepsilon$ de $I$ pour lesquels il existe des indices $i, j \in \llbracket 1, s \rrbracket$ tels que

$$
\forall \varepsilon^{\prime} \in I \cap\left[0, \varepsilon\left[, \quad C_{i, \beta} \cap C_{j, \beta} \cap V_{\varepsilon^{\prime}} \neq \emptyset\right.\right.
$$

et

$$
\left.\forall \varepsilon^{\prime} \in I \cap\right] \varepsilon,+\infty\left[, \quad C_{i, \beta} \cap C_{j, \beta} \cap V_{\varepsilon^{\prime}}=\emptyset .\right.
$$

En d'autres termes, chaque élément de $E$ peut être obtenu comme la valeur maximale de la valeur absolue de la fonction $f$ sur un certain domaine strictement affinode de $X$. D'après [BGR84, 6.2.1/4], on en déduit que $E \subset \sqrt{\left|k^{*}\right|}$.

Tâchons, tout d'abord, de démontrer qu'il existe une partition finie de $\mathbb{R}^{+}$en intervalles réguliers pour $f$ sur $X$ et dont les bornes sont des éléments de $\sqrt{\left|k^{*}\right|} \cup\{0,+\infty\}$. Puisque l'espace $k$-affinoïde $X$ possède un nombre fini de composantes irréductibles, le lemme précédent nous montre qu'il suffit de le prouver pour chacune d'elles. Nous pouvons donc supposer que l'espace $X$ est irréductible et même intègre. D'après [BGR84, 6.2.1/4 (ii)], si la fonction $f$ n'est pas nulle, nous pouvons supposer que sa norme spectrale vaut 1 . Dans ce cas, nous savons, d'après le théorème 1, qu'il existe $\left.\left.\varepsilon^{\prime} \in\right] 0,1\right] \cap \sqrt{\left|k^{*}\right|}$ tel que, quel que soit $\left.\left.\varepsilon \in\right] 0, \varepsilon^{\prime}\right]$, l'espace

$$
V_{\varepsilon}=\{x \in X|| f(x) \mid \geqslant \varepsilon\}
$$




\section{J. POINEAU}

soit connexe. Par hypothèse, l'espace $V_{0}=X$ est connexe, donc l'intervalle $\left[0, \varepsilon^{\prime}\right.$ [ est régulier pour $f$ sur $X$. Quel que soit $\varepsilon \in] 1,+\infty\left[\right.$, l'espace $V_{\varepsilon}$ est vide et l'intervalle ]1, $+\infty$ [ est donc également régulier pour $f$ sur $X$. Le théorème 1.10 nous assure encore qu'il est possible de découper l'intervalle $\left[\varepsilon^{\prime}, 1\right]$ en un nombre fini d'intervalles réguliers pour $f$ sur $X$ et dont les bornes sont des éléments de $\sqrt{\left|k^{*}\right|} \cup\{0,+\infty\}$. Par conséquent, il existe une partition finie de $\mathbb{R}^{+}$en intervalles réguliers pour $f$ sur $X$ dont les bornes jouissent de la même propriété.

Intéressons-nous à présent à la forme des intervalles de la partition précédente. Les lemmes qui suivent nous permettront de l'obtenir, concluant ainsi la démonstration du théorème 2 .

Lemme 4.5. Quel que soit $\beta>0$, il existe $\alpha \in[0, \beta[$ tel que, quel que soit $\varepsilon \in[\alpha, \beta]$, l'application naturelle

$$
\pi_{0}\left(V_{\beta}\right) \rightarrow \pi_{0}\left(V_{\varepsilon}\right)
$$

soit injective.

Démonstration. Soit $\beta>0$. Puisque $V_{\beta}$ ne possède qu'un nombre fini de composantes connexes, il suffit de montrer que deux d'entre elles, $C_{0}$ et $C_{1}$, distinctes, sont contenues dans deux composantes connexes distinctes de $V_{\varepsilon}$, avec $\varepsilon \leqslant \beta$, dès que $\varepsilon$ est assez proche de $\beta$.

Remarquons qu'il existe une fonction, $g$ analytique sur $V_{\beta}$, vérifiant

$$
g_{\mid C_{0}} \equiv 0, g_{\mid C_{1}} \equiv 1 \quad \text { et } \quad g^{2}-g=0 .
$$

Puisque $V_{\beta}$ est un domaine rationnel de $X$, nous pouvons approcher la fonction $g$, uniformément sur $V_{\beta}$, par une suite de quotients d'éléments de $\mathscr{A}$ sans pôles sur $V_{\beta}$. Par conséquent, il existe $p, q \in \mathscr{A}$ tels que $q$ ne s'annule pas sur $V_{\beta}$ et

$$
\forall x \in V_{\beta}, \quad|g(x)-h(x)| \leqslant \frac{1}{3} \quad \text { et } \quad\left|h^{2}(x)-h(x)\right| \leqslant \frac{1}{5},
$$

où $h=p / q$. Le lieu d'annulation de $q$ est une partie compacte de $X$, disjointe de $V_{\beta}$, sur laquelle la fonction continue $f$ atteint son maximum $M<\beta$. Soit $\left.\left.M^{\prime} \in\right] M, \beta\right]$. La fonction méromorphe $h$ est analytique sur $V_{M^{\prime}}$. Définissons un compact $K$ de $V_{M^{\prime}}$ par

$$
K=\left\{x \in V_{M^{\prime}}|| h^{2}(x)-h(x) \mid \geqslant \frac{1}{4}\right\} .
$$

La fonction continue $f$ y atteint son maximum $M_{1}$. Puisque $K$ et $V_{\beta}$ sont disjoints, on a nécessairement $M_{1}<\beta$. Fixons $\left.\alpha \in\right] M_{1}, \beta[$.

Soit $\varepsilon \in[\alpha, \beta]$. Le compact $K$ est disjoint de $V_{\varepsilon}$ donc, quel que soit $x \in V_{\varepsilon}$, on a $|h(h-1)(x)|<$ 1/4. Posons

$$
D_{0}=\left\{x \in V_{\varepsilon}|| h(x) \mid<\frac{1}{2}\right\} \quad \text { et } \quad D_{1}=\left\{x \in V_{\varepsilon}|| h(x)-1 \mid<\frac{1}{2}\right\} .
$$

Ces deux ouverts sont disjoints et recouvrent $V_{\varepsilon}$. Par conséquent, ils sont réunions de composantes connexes. En outre, $C_{0} \subset D_{0}$ et $C_{1} \subset D_{1}$, donc les parties $C_{0}$ et $C_{1}$ sont contenues dans deux composantes connexes distinctes de $V_{\varepsilon}$.

Lemme 4.6. Si l'intervalle $[\alpha, \beta$ [ est régulier pour $f$ sur $X$, alors l'intervalle $[\alpha, \beta]$ l'est encore.

Démonstration. Il nous suffit de montrer que l'application naturelle

$$
\iota: \pi_{0}\left(V_{\beta}\right) \rightarrow \pi_{0}\left(V_{\alpha}\right)
$$

est bijective. D'après le lemme 4.5, elle est injective. Montrons qu'elle est également surjective.

Soit $C$ une composante connexe de $V_{\alpha}$. C'est une partie compacte sur laquelle la fonction continue $f$ atteint son maximum $M$. Puisque l'intervalle $\left[\alpha, \beta\left[\right.\right.$ est régulier pour $f$ sur $X$, on a $C \cap V_{\varepsilon} \neq \emptyset$ et donc $M \geqslant \varepsilon$, quel que soit $\varepsilon \in\left[\alpha, \beta\left[\right.\right.$. On en déduit que $M \geqslant \beta$, autrement dit que $C \cap V_{\beta} \neq \emptyset$. Choisissons une composante connexe de $V_{\beta}$ coupant $C$. Elle s'envoie sur $C$ par $\iota$. L'application $\iota$ est donc surjective. 


\section{ConneXITÉ EN GÉOMÉTRIQUE ANALYTIQUe $p$-ADIQUE}

Il nous reste à démontrer la partie du théorème 2 qui concerne les composantes irréductibles. Elle se déduit de celle qui concerne les composantes connexes lorsqu'on l'applique au normalisé de $X$.

Énonçons, à présent, un corollaire du théorème 1. Il figure déjà dans [Berk90, § 2.3], sans démonstration.

Corollaire 4.7. Un point d'un bon espace $k$-analytique en lequel l'anneau local est intègre possède une base de voisinages affinoïdes irréductibles.

Démonstration. L'espace étant bon, il suffit de démontrer que tout point d'un espace $k$-affinoïde en lequel l'anneau local est intègre possède un voisinage affinoïde irréductible. Soient $Y$ un espace $k$-affinoïde et $y$ un point de $Y$ en lequel l'anneau local $\mathscr{O}_{Y, y}$ est intègre. Alors le point $y$ ne peut être situé que sur une seule des composantes irréductibles de $Y$. Notons $F$ cette composante et $G$ la réunion des autres. Soit $g$ une fonction analytique sur $Y$ nulle sur le fermé de Zariski $G$ et ne s'annulant pas en $y$. D'après le théorème 1 , il existe $\varepsilon \in] 0,|f(x)|[$ tel que le domaine affinoïde

$$
\left\{y^{\prime} \in Y|| f\left(y^{\prime}\right) \mid \geqslant \varepsilon\right\}=\left\{y^{\prime} \in F|| f\left(y^{\prime}\right) \mid \geqslant \varepsilon\right\}
$$

soit irréductible.

\section{Privilège}

Dans cette partie, nous énonçons et démontrons un résultat de privilège pour les variétés analytiques $p$-adiques. La section 7 de l'article [Dou66] de Douady est consacrée à cette notion, dans le cadre analytique complexe. Rappelons-en quelques définitions et notations.

Si $K$ est une partie compacte de $\mathbb{C}^{n}$, avec $n \in \mathbb{N}$, on note $\mathscr{O}(K)$ l'espace vectoriel des germes de fonctions analytiques au voisinage de $K$ et $B(K)$ son adhérence dans l'espace de Banach des fonctions continues sur $K$. Si $\mathscr{F}$ est un faisceau analytique cohérent défini au voisinage de $K$, on note $\mathscr{F}(K)$ la limite inductive des modules des sections de $\mathscr{F}$ sur les voisinages ouverts de $K$ et

$$
B(K, \mathscr{F})=B(K) \otimes_{\mathscr{O}(K)} \mathscr{F}(K) .
$$

Revenons, à présent, au cadre des espaces analytiques définis sur un corps ultramétrique complet. Soient $Y$ un espace $k$-analytique normal et séparé et $V=\mathscr{M}(\mathscr{A})$ un domaine affinoïde de $Y$ contenu dans l'intérieur de $Y$. En définissant $B(V)$ de la même façon que précédemment, on obtient un isomorphisme

$$
B(V) \stackrel{\sim}{\longrightarrow} \mathscr{A}
$$

et l'on retrouve les sections du faisceau structural pour la G-topologie. De même, si $\mathscr{F}$ désigne un faisceau cohérent pour la G-topologie de $Y$, la formule définissant $B(V, \mathscr{F})$ redonne exactement le $\mathscr{A}$-module de type fini $\mathscr{F}(V)$ des sections globales de $\mathscr{F}$ sur $V$.

DÉFINition 5.1. Soient $k$ un corps ultramétrique complet, $Y$ un bon espace $k$-analytique et $\mathscr{F}$ un faisceau cohérent défini sur $Y$. Nous dirons qu'un voisinage affinoïde $V$ d'un point $y$ de l'espace $k$-analytique $Y$ est privilégié pour le faisceau $\mathscr{F}$ s'il vérifie

$$
\mathscr{F}(V) \hookrightarrow \mathscr{F}_{y},
$$

où $\mathscr{F}(V)$ doit être pris au sens de la $G$-topologie et $\mathscr{F}_{y}$ au sens de la topologie sur l'espace topologique sous-jacent $|Y|$.

Pour qu'un voisinage compact $K$ d'un point soit privilégié, Douady impose non seulement la condition qui figure dans la définition, mais encore une autre qui porte sur des propriétés d'exactitude du foncteur $B(K, \cdot)$ (cf. [Dou66, $\S 7$, définition 2]). Dans notre cadre, elles seront toujours vérifiées pour les domaines affinoïdes. 


\section{J. Poineau}

Signalons que l'on peut penser à un voisinage privilégié pour un faisceau cohérent comme un voisinage sur lequel vaut une généralisation de l'unicité du prolongement analytique. En effet, si $Y$ désigne un espace $k$-analytique irréductible et réduit, nous savons, d'après [Berk90, 3.3.21], qu'une fonction nulle sur un ouvert non vide de $Y$ est identiquement nulle. Ce résultat se traduit par le fait que $Y$ est un voisinage privilégié de tous ses points pour le faisceau structural. Nous en déduisons aussitôt le lemme suivant.

Lemme 5.2. Dans un espace $k$-analytique réduit, un voisinage affinoïde d'un point est privilégié pour le faisceau structural dès que toutes les composantes irréductibles du voisinage passent par ce point.

Nous souhaitons montrer ici que tout point d'un espace $k$-analytique possède un système fondamental de voisinages affinoïdes privilégiés pour un faisceau cohérent fixé, du moins lorsque l'espace est bon. Ce résultat est analogue à celui démontré par Douady dans [Dou66, §6, théorème 1]. Notre démonstration reprend des idées qui figurent dans l'article [Fri67] de Frisch.

LEMme 5.3. Soient $Y$ un espace $k$-analytique, $y$ un point de $Y$ et

$$
0 \rightarrow \mathscr{F}^{\prime} \rightarrow \mathscr{F} \rightarrow \mathscr{F}^{\prime \prime}
$$

une suite exacte de faisceaux cohérents sur $Y$. Alors, un voisinage affinö̈de de y privilégié pour les faisceaux $\mathscr{F}^{\prime}$ et $\mathscr{F}^{\prime \prime}$ l'est encore pour le faisceau $\mathscr{F}$.

Démonstration. Ce résultat provient directement de l'exactitude à gauche du foncteur des sections globales.

Lemme 5.4. Soient $Y$ un espace $k$-affinoïde et $\mathscr{F}$ un faisceau cohérent sur $Y$. Alors il existe un entier $r \in \mathbb{N}$, une filtration

$$
0=\mathscr{F}_{0} \subset \mathscr{F}_{1} \subset \cdots \subset \mathscr{F}_{r}=\mathscr{F}
$$

de $\mathscr{F}$ par des sous-faisceaux cohérents et $r$ fermés de Zariski de $Y$ intègres $Z_{0}, \ldots, Z_{r-1}$ vérifiant la condition suivante: quel que soit $i \in \llbracket 0, r-1 \rrbracket$, on dispose d'un isomorphisme de faisceaux

$$
\mathscr{F}_{i+1} / \mathscr{F}_{i} \simeq \mathscr{O}_{Z_{i}} \text {. }
$$

Démonstration. Le module $\mathscr{F}(Y)$ des sections du faisceau cohérent $\mathscr{F}$ sur $Y$ est un module de type fini sur l'algèbre $\mathscr{B}$ de $Y$. Par conséquent, il existe un entier $r \in \mathbb{N}$, une filtration

$$
0=M_{0} \subset M_{1} \subset \cdots \subset M_{r}=M
$$

de $M$ par des sous- $\mathscr{B}$-modules de type fini vérifiant la condition suivante $:$ quel que soit $i \in \llbracket 0, r-1 \rrbracket$, il existe un idéal premier $\mathfrak{p}_{i}$ de $\mathscr{B}$ et un isomorphisme

$$
M_{i+1} / M_{i} \simeq \mathscr{B} / \mathfrak{p}_{i} .
$$

Pour $i \in \llbracket 0, r \rrbracket$, notons $\mathscr{F}_{i}$ le faisceau cohérent associé à $M_{i}$ sur $Y$ et, pour $i \in \llbracket 0, r-1 \rrbracket$, notons $Z_{i}$ le fermé de Zariski intègre de $Y$ d'algèbre $\mathscr{B} / \mathfrak{p}_{i}$. Ils satisfont la conclusion du lemme.

ThÉorÈme 5.5. Soient $k$ un corps ultramétrique complet, $Y$ un bon espace $k$-analytique et $\mathcal{F}$ une famille finie de faisceaux cohérents sur $Y$. Tout point de $Y$ possède un système fondamental de voisinages affinoïdes privilégiés pour chacun des faisceaux de $\mathcal{F}$.

Démonstration. Soit $y \in Y$. Par définition d'un bon espace, le point $y$ possède un système fondamental $\mathscr{V}$ de voisinages affinoïdes dans $Y$. Il suffit de montrer que, quel que soit $V \in \mathscr{V}$, le point $y$ possède un voisinage affinoïde dans $V$ qui soit privilégié pour chacun des faisceaux de $\mathcal{F}$. Soit $V \in \mathscr{V}$. Notons $\mathscr{B}$ son algèbre. 


\section{ConneXITÉ EN GÉOMÉTRIQUE ANALYTIQUe $p$-ADIQUE}

Soit $\mathscr{F}$ un élément de $\mathcal{F}$. D'après le lemme 5.4, il existe entier $r(\mathscr{F}) \in \mathbb{N}$, une filtration

$$
0=\mathscr{F}_{0} \subset \mathscr{F}_{1} \subset \cdots \subset \mathscr{F}_{r(\mathscr{F})}=\mathscr{F}
$$

de $\mathscr{F}$ par des sous-faisceaux cohérents et $r(\mathscr{F})$ fermés de Zariski de $V$ intègres

$$
Z_{\mathscr{F}, 0}, \ldots, Z_{\mathscr{F}, r(\mathscr{F})-1}
$$

vérifiant la condition suivante: quel que soit $i \in \llbracket 0, r(\mathscr{F})-1 \rrbracket$, on dispose d'un isomorphisme de faisceaux

$$
\mathscr{F}_{i+1} / \mathscr{F}_{i} \simeq \mathscr{O}_{Z_{\mathscr{F}, i}} .
$$

Définissons l'ensemble

$$
\mathcal{P}=\left\{Z_{\mathscr{F}, i}, \mathscr{F} \in \mathcal{F}, 0 \leqslant i \leqslant r(\mathscr{F})-1\right\} .
$$

D'après le lemme 5.3, il nous suffit pour conclure de montrer que le point $y$ possède un voisinage affinoïde privilégié pour chacun des faisceaux $\mathscr{O}_{Z}$, avec $Z \in \mathcal{P}$. Notons $\mathcal{Q}$ l'ensemble des éléments de $\mathcal{P}$ évitant le point $y$. Leur réunion $R$ définit un fermé de Zariski de $V$ ne contenant pas $y$. Par conséquent, il existe une fonction $g \in \mathscr{B}$ qui soit nulle sur $R$, mais pas en $y$. D'après le théorème 1 , il existe $\varepsilon \in] 0,|g(y)|[$ tel que l'espace affinoïde

$$
\{z \in Z|| g(z) \mid \geqslant \varepsilon\}
$$

soit irréductible, quel que soit $Z$ dans $\mathcal{P} \backslash \mathcal{Q}$. Notons $W$ le voisinage affinoïde de $y$ dans $V$ défini par

$$
W=\{z \in V|| g(z) \mid \geqslant \varepsilon\} .
$$

Soit $Z \in \mathcal{P}$. Si $Z \in \mathcal{Q}$, le faisceau $\mathscr{O}_{Z}$ restreint à $W$ est nul et le voisinage $W$ de $y$ est donc privilégié pour $\mathscr{O}_{Z}$. Si $Z \notin \mathcal{Q}$, le fermé de Zariski $Z \cap W$ est irréductible et on dispose donc d'un morphisme injectif

$$
\mathscr{O}_{Z}(W) \simeq \mathscr{O}_{Z \cap W}(Z \cap W) \hookrightarrow \mathscr{O}_{Z, y}
$$

Autrement dit, le voisinage $W$ de $y$ est, dans ce cas encore, privilégié pour $\mathscr{O}_{Z}$.

\section{Appendice A. Un analogue p-adique du théorème de Frisch}

Nous proposons ici un analogue, dans le cadre des espaces analytiques définis sur un corps ultramétrique complet, du théorème I,9 qui figure dans l'article [Fri67] de Frisch. Nous suivrons, ici, la démonstration de Bănică et Stănăşilă [BS77, chapitre 5, fin de la section 3]. Nous obtiendrons une version un peu plus générale du théorème, proche de celle que propose Siu dans [Siu69]. Commençons par une définition et un lemme.

DÉfinition A.1. Soient $k$ un corps ultramétrique complet et $Y$ un espace $k$-analytique. Une partie $A$ de $Y$ est dite morcelable si, pour tout fermé de Zariski $Z$ défini au voisinage de $A$, l'image réciproque de $A \cap Z$ dans le normalisé de $Z$ possède un nombre fini de composantes connexes.

Lemme A.2. Soient $k$ un corps ultramétrique complet et $Y$ un espace $k$-analytique. Si l'espace $Y$ est normal, alors le support de tout faisceau d'idéaux cohérent sur $Y$ est ouvert et fermé.

Démonstration. Nous pouvons supposer que $Y$ est un espace $k$-affinoïde. Soit $\mathscr{J}$ un faisceau d'idéaux cohérents sur $Y$. Comme tout faisceau cohérent, son support est fermé dans $Y$. Pour conclure, il nous suffit de montrer qu'il est également ouvert. Soit $y$ un point de $Y$ en lequel la fibre de $\mathscr{J}$ n'est pas nulle. Alors il existe un voisinage ouvert connexe $V$ de $y$ et une fonction analytique $g \in \mathscr{J}(V)$ qui ne soit pas identiquement nulle sur $V$. Soit $z \in V$. D'après [Berk90, 3.3.21], le fermé de Zariski défini par $g$ est d'intérieur vide dans l'espace normal et connexe $V$. En particulier, la fonction $g$ n'est nulle sur aucun voisinage de $z$ dans $V$. On en déduit que la fibre $\mathscr{J}_{z}$ n'est pas nulle et donc que le support de $\mathscr{J}$ est ouvert. 


\section{J. POINEAU}

Le résultat de finitude sur lequel nous nous appuierons concerne les familles croissantes de faisceaux cohérents.

Lemme A.3. Soient $k$ un corps ultramétrique complet et $Y$ un espace $k$-analytique. Notons $\pi: \tilde{Y} \rightarrow$ $Y$ le morphisme de normalisation. Soit $A$ une partie de l'espace topologique sous-jacent à $Y$ telle que $\pi^{-1}(A)$ possède un nombre fini de composantes connexes. Soit $\left(\mathscr{I}_{n}\right)_{n \in \mathbb{N}}$ une suite croissante de faisceaux d'idéaux cohérents de $\mathscr{O}_{Y}$ définis chacun sur un voisinage de $A$. Soit a un point de $A$ en lequel la fibre $\left(\mathscr{I}_{n}\right)_{a}$ est nulle, quel que soit $n \in \mathbb{N}$. Alors il existe un voisinage de a dans $A$ sur lequel toutes les fibres du faisceau $\mathscr{I}_{n}$ sont nulles, quel que soit $n \in \mathbb{N}$.

Démonstration. Quel que soit $b \in \pi^{-1}(a)$, il existe un voisinage $V_{b}$ de $b$ dans $\tilde{Y}$ tel que $\pi^{-1}(A) \cap V_{b}$ soit connexe. La partie $V=\bigcup_{b \in \pi^{-1}(A)} V_{b}$ définit un voisinage de la fibre $\pi^{-1}(a)$ dans $\tilde{Y}$, donc il existe un voisinage $U$ de $a$ dans $Y$ tel que $\pi^{-1}(U) \subset V$. Nous allons montrer que, quel que soit $n \in \mathbb{N}$ et quel que soit $a^{\prime} \in U \cap A$, on a

$$
\left(\mathscr{I}_{n}\right)_{a^{\prime}}=0 .
$$

Soit $n \in \mathbb{N}$. Notons $\mathscr{J}_{n}$ le faisceau défini par

$$
\mathscr{J}_{n}=\pi^{-1}\left(\mathscr{I}_{n}\right) \mathscr{O}_{\tilde{Y}}
$$

C'est un faisceau d'idéaux cohérent défini sur un voisinage de $\pi^{-1}(A)$ dans $\tilde{Y}$. Quel que soit $b \in$ $\pi^{-1}(a)$, il existe un voisinage $V_{b, n}$ de $\pi^{-1}(A) \cap V_{b}$ sur lequel $\mathscr{J}_{n}$ est défini. Puisque

$$
\left(\mathscr{J}_{n}\right)_{b}=\left(\mathscr{I}_{n}\right)_{a} \mathscr{O}_{\tilde{Y}, b}=0,
$$

la fibre de $\mathscr{J}_{n}$ est nulle en tout point de $V_{b, n}$, d'après le lemme A.2. La partie $V_{n}=\bigcup_{b \in \pi^{-1}(A)} V_{b, n}$ est un voisinage de $\pi^{-1}(A \cap U)$ dans $\tilde{Y}$. Par conséquent, la fibre du faisceau $\pi_{*} \mathscr{J}_{n}$ est nulle en tout point de $A \cap U$. Or le diagramme commutatif

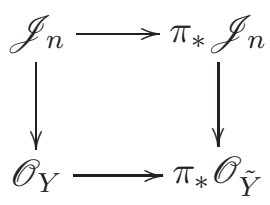

montre que le faisceau $\mathscr{J}_{n}$ s'injecte dans le faisceau $\pi_{*} \mathscr{J}_{n}$. Le résultat annoncé s'en déduit.

Proposition A.4. Soient $k$ un corps ultramétrique complet, $Y$ un espace $k$-affinoïde et $A$ une partie de l'espace topologique sous-jacent à $Y$. Soient $\mathscr{F}$ un faisceau cohérent défini sur $Y$ et $\left(\mathscr{F}_{n}\right)_{n \in \mathbb{N}}$ une suite croissante de sous-faisceaux cohérents de $\mathscr{F}$ définis chacun sur un voisinage affinoïde de A. Si la partie $A$ est morcelable, alors la suite $\left(\mathscr{F}_{n}\right)_{n \in \mathbb{N}}$ est localement stationnaire dans $A$ au sens où, quel que soit $a \in A$, il existe un entier $n_{0} \in \mathbb{N}$ et un voisinage $U$ de $a$ dans $A$ tels que

$$
\forall n \geqslant n_{0}, \quad \forall z \in A, \quad\left(\mathscr{F}_{n_{0}}\right)_{z} \stackrel{\sim}{\longrightarrow}\left(\mathscr{F}_{n}\right)_{z} .
$$

Démonstration. Supposons que la partie $A$ soit morcelable. Soit $a \in A$. Il existe $n_{0} \in \mathbb{N}$ tel que, quel que soit $n \geqslant n_{0}$, on ait

$$
\left(\mathscr{F}_{n_{0}}\right)_{a} \stackrel{\sim}{\longrightarrow}\left(\mathscr{F}_{n}\right)_{a} .
$$

Quitte à restreindre $Y$, à remplacer $\mathscr{F}$ par $\mathscr{F} / \mathscr{F}_{n_{0}}$ et $\mathscr{F}_{n}$ par $\mathscr{F}_{n} / \mathscr{F}_{n_{0}}$, pour $n \geqslant n_{0}$, puis à décaler les indices, nous pouvons supposer que

$$
\left(\mathscr{F}_{n}\right)_{a}=0,
$$

quel que soit $n \in \mathbb{N}$. D'après le lemme 5.4, il existe un entier $r \in \mathbb{N}$, une filtration

$$
0=\mathscr{F}^{(0)} \subset \mathscr{F}^{(1)} \subset \cdots \subset \mathscr{F}^{(r)}=\mathscr{F}
$$


de $\mathscr{F}$ par des sous-faisceaux cohérents et $r$ fermés de Zariski de $Y$ intègres $Z_{0}, \ldots, Z_{r-1}$ vérifiant la condition suivante: quel que soit $i \in \llbracket 0, r-1 \rrbracket$, on dispose d'un isomorphisme de faisceaux

$$
\mathscr{F}^{(i+1)} / \mathscr{F}^{(i)} \simeq \mathscr{O}_{Z_{i}}
$$

Il nous suffit, à présent, de montrer que, pour chaque $i \in \llbracket 0, r-1 \rrbracket$, la sous-suite $\left(\mathscr{G}_{i, n}\right)_{n \in \mathbb{N}}$ de $\mathscr{F}^{(i)} / \mathscr{F}^{(i+1)} \simeq \mathscr{O}_{Z_{i}}$ induite par $\left(\mathscr{F}_{n}\right)_{n \in \mathbb{N}}$ stationne au voisinage de $a$ dans $A$ et même au voisinage de $a$ dans $A \cap Z_{i}$. Le lemme précédent nous permet de conclure.

Il ne nous reste plus qu'à rendre global le résultat précédent pour obtenir le théorème recherché.

ThÉorème A.5. Soient $k$ un corps ultramétrique complet, $Y$ un bon espace $k$-analytique et $K$ une partie compacte de l'espace topologique sous-jacent à $Y$. Si $K$ est morcelable et possède un système fondamental de voisinages affinoïdes, alors l'anneau $\mathscr{O}(Y, K)$ des germes de fonctions analytiques au voisinage de $K$ est noethérien.

Démonstration. Soit $\left(I_{n}\right)_{n \in \mathbb{N}}$ une suite croissante d'idéaux de type fini de $\mathscr{O}(Y, K)$. Pour $n \in \mathbb{N}$, notons $\mathscr{I}_{n}$ le faisceau d'idéaux cohérents de $\mathscr{O}_{Y}$ engendré par $I_{n}$. D'après la proposition précédente, la suite $\left(\mathscr{I}_{n}\right)_{n \in \mathbb{N}}$ stationne sur $K$, au sens où il existe $n_{0} \in \mathbb{N}$ tel que, quel que soit $n \geqslant n_{0}$ et quel que soit $y \in K$, on dispose d'un isomorphisme

$$
\left(\mathscr{I}_{n_{0}}\right)_{y} \stackrel{\sim}{\longrightarrow}\left(\mathscr{I}_{n}\right)_{y} .
$$

Puisque l'idéal $I_{n_{0}}$ est fini, il possède un système générateur fini $\left(f_{1}, \ldots, f_{p}\right)$, avec $p \in \mathbb{N}$ et $f_{i} \in \mathscr{O}(Y, K)$, quel que soit $i \in \llbracket 1, p \rrbracket$. Le morphisme de faisceaux

$$
\varphi: \begin{array}{ccc}
\mathscr{O}_{Y}^{p} & \rightarrow & \mathscr{I}_{n_{0}} \\
\left(a_{1}, \ldots, a_{p}\right) & \mapsto & a_{1} f_{1}+\cdots+a_{p} f_{p}
\end{array}
$$

est surjectif.

Soit $n \geqslant n_{0}$. Si un morphisme entre deux faisceaux cohérents induit un isomorphisme entre les fibres en un point, alors il induit un isomorphisme au voisinage de ce point. Par conséquent, les faisceaux $\mathscr{I}_{n_{0}}$ et $\mathscr{I}_{n}$ coïncident sur un voisinage $U_{n}$ de $K$. Soient $g \in I_{n}$ et $V_{n}$ un voisinage affinoïde de $K$ dans $U_{n}$ sur lequel les fonctions $f_{1}, \ldots, f_{p}, g$ soient définies. Notons $\mathscr{G}$ le noyau du morphisme de faisceaux $\varphi$. C'est encore un faisceau cohérent sur $V_{n}$. De la suite exacte $0 \rightarrow \mathscr{G} \rightarrow \mathscr{O}^{p} \rightarrow \mathscr{I}_{n} \rightarrow 0$, on déduit une surjection

$$
\begin{array}{ccc}
\mathscr{O}\left(V_{n}\right)^{p} & \rightarrow & \mathscr{I}_{n}\left(V_{n}\right), \\
\left(a_{1}, \ldots, a_{p}\right) & \mapsto & a_{1} f_{1}+\cdots+a_{p} f_{p},
\end{array}
$$

car $H^{1}\left(V_{n}, \mathscr{G}\right)=0$. Par conséquent,

$$
g \in\left(f_{1}, \ldots, f_{p}\right) \mathscr{O}\left(V_{n}\right) \subset\left(f_{1}, \ldots, f_{p}\right) \mathscr{O}(Y, K)=I_{n_{0}} .
$$

On en déduit que $I_{n}=I_{n_{0}}$.

Signalons que le théorème que démontre Frisch concerne des compacts possédant un système fondamental de voisinages composé d'espaces de Stein. Dans le cadre des espaces analytiques définis sur un corps ultramétrique complet $k$, il existe également une notion d'espace de Stein. Un espace $k$-analytique $Y$ est dit de Stein s'il existe une suite croissante $\left(Y_{n}\right)_{n \in \mathbb{N}}$ de domaines affinoïdes de $Y$ vérifiant les conditions suivantes :

(a) quel que soit $n \in \mathbb{N}, Y_{n}$ est un domaine de Weierstraß de $Y_{n+1}$;

(b) la famille $\left\{Y_{n}, n \in \mathbb{N}\right\}$ définit un G-recouvrement de $Y$.

La seconde condition, présente dans [Kie67, 2.3], fait défaut dans [Berk90, p. 96]. 


\section{J. POINEAU}

Il découle de la définition qu'une partie compacte d'un espace analytique possédant un système fondamental de voisinages constitué d'espaces de Stein possède encore un système fondamental de voisinages constitué d'affinoïdes.

Mentionnons, pour conclure, deux exemples de parties compactes morcelables:

(a) Nous dirons qu'une partie $A$ d'un espace analytique $Y$ est semi-analytique si tout point de $A$ possède un voisinage affinoïde dans lequel la partie $A$ est semi-algébrique, c'est-à-dire décrite par un nombre fini d'inégalités entre fonctions. Une telle partie est morcelable, lorsqu'elle est compacte, d'après [Duc03c, 3.2]. Dans ce cas, nous retrouvons exactement l'énoncé original de Frisch.

(b) Si $Y$ est un espace $k$-analytique et $K$ une extension de $k$, nous noterons $Y_{K}$ l'espace $K$ analytique obtenu par extension du corps de base. Nous dirons qu'un morphisme $\varphi$ entre espaces $k$-analytiques est une immersion s'il se décompose sous la forme

$$
\varphi: Z \hookrightarrow Y_{K} \rightarrow Y,
$$

où $Z$ désigne un fermé de Zariski d'un domaine analytique de $Y_{K}$ et s'il induit un homéomorphisme de $Z$ sur son image $Z^{\prime}$ et des isomorphismes entre les corps résiduels complétés en tous les points de $Z^{\prime}$. L'image de toute immersion définit une partie morcelable. Les fibres des morphismes entre espaces $k$-analytiques rentrent, par exemple, dans ce cadre. Remarquons qu'elles ne sont pas semi-analytiques, en général.

\section{REMERCIEMENTS}

Je tiens à remercier Antoine Chambert-Loir pour les nombreux conseils qu'il m'a prodigués. C'est sur ses indications que je me suis intéressé au théorème de H. Epp, sans lequel ce travail n'aurait pu être mené à terme. Ma gratitude va également à Antoine Ducros pour avoir suivi avec attention l'avancée de mes recherches et avoir toujours accepté de partager avec moi sa passion pour les espaces de Berkovich. J'exprime également mes remerciements à Ahmed Abbes pour l'intérêt qu'il a porté à mon travail et ses remarques qui m'ont permis de préciser le résultat du théorème 2 . Finalement, je sais gré à Qing Liu qui a lu attentivement ce texte et m'a invité à étendre le résultat du théorème 1 .

\section{REFERENCES}

AS02 A. Abbes and T. Saito, Ramification of local fields with imperfect residue fields, Amer. J. Math. 124 (2002), 879-920.

BS77 C. Bănică and O. Stănăşilă, Méthodes algébriques dans la théorie globale des espaces complexes, Vol. 2, Troisième édition, in Traduit du roumain, Collection Varia Mathematica (Gauthier-Villars, Paris, 1977).

Bar70 W. Bartenwerfer, Einige Fortsetzungssätze in der p-adischen Analysis, Math. Ann. 185 (1970), $191-210$.

Berk90 V. G. Berkovich, Spectral theory and analytic geometry over non-Archimedean fields, Mathematical Surveys and Monographs, vol. 33 (American Mathematical Society, Providence, RI, 1990).

Berk93 V. G. Berkovich, Étale cohomology for non-Archimedean analytic spaces, Publ. Math. Inst. Hautes Études Sci. 78 (1993), 5-161.

Berk94 V. G. Berkovich, Vanishing cycles for formal schemes, Invent. Math. 115 (1994), 539-571.

Berk99 V. G. Berkovich, Smooth p-adic analytic spaces are locally contractible, Invent. Math. 137 (1999), $1-84$.

Berk04 V. G. Berkovich, Smooth p-adic analytic spaces are locally contractible. II, in Geometric aspects of Dwork theory, Vol. I, II (Walter de Gruyter, Berlin, 2004), 293-370. 


\section{ConneXITÉ EN GÉOMÉTRIQUE ANALYTIQUe $p$-ADIQUE}

Bert96 P. Berthelot, Cohomologie rigide et cohomologie rigide à supports propres, première partie, Prépublication de l'IRMAR 96-03 (1996),

http://perso.univ-rennes1.fr/pierre.berthelot/publis/Cohomologie_Rigide_I.pdf.

BGR84 S. Bosch, U. Güntzer and R. Remmert, Non-Archimedean analysis: a systematic approach to rigid analytic geometry, Grundlehren der Mathematischen Wissenschaften, vol. 261 (Springer, Berlin, 1984).

BL93a S. Bosch and W. Lütkebohmert, Formal and rigid geometry. I. Rigid spaces, Math. Ann. 295 (1993), 291-317.

BL93b S. Bosch and W. Lütkebohmert, Formal and rigid geometry. II. Flattening techniques, Math. Ann. 296 (1993), 403-429.

Bos95a S. Bosch, W. Lütkebohmert and M. Raynaud, Formal and rigid geometry. III. The relative maximum principle, Math. Ann. 302 (1995), 1-29.

Bos95b S. Bosch, W. Lütkebohmert and M. Raynaud, Formal and rigid geometry. IV. The reduced fibre theorem, Invent. Math. 119 (1995), 361-398.

Dou66 A. Douady, Le problème des modules pour les sous-espaces analytiques compacts d'un espace analytique donné, Ann. Inst. Fourier (Grenoble) 16 (1996), 1-95.

Duc03a A. Ducros, Étude de certaines propriétés locales et globales des espaces de Berkovich, Prépublication de l'IRMAR 03-41 (2003), http://math.unice.fr/ ducros/geoanabis.pdf.

Duc03b A. Ducros, Image réciproque du squelette par un morphisme entre espaces de Berkovich de même dimension, Bulletin de la Société Mathématique de France, No. 131 (Société Mathématique de France, Paris, 2003), 483-506.

Duc03c A. Ducros, Parties semi-algébriques d'une variété algébrique p-adique, Manuscripta Math. 111 (2003), 513-528.

Epp73 H. P. Epp, Eliminating wild ramification, Invent. Math. 19 (1973), 235-249.

Fri67 J. Frisch, Points de platitude d'un morphisme d'espaces analytiques complexes, Invent. Math. 4 (1967), 118-138.

Gro64 A. Grothendieck, Éléments de géométrie algébrique. IV. Étude locale des schémas et des morphismes de schémas. I, Publ. Math. Inst. Hautes Études Sci. 20 (1964), 259.

Gro65 A. Grothendieck, Éléments de géométrie algébrique. IV. Étude locale des schémas et des morphismes de schémas. II, Publ. Math. Inst. Hautes Études Sci. 24 (1965), 231.

Gro66 A. Grothendieck, Éléments de géométrie algébrique. IV. Étude locale des schémas et des morphismes de schémas. III, Publ. Math. Inst. Hautes Études Sci. 28 (1966), 255.

Kie67 R. Kiehl, Theorem A und Theorem B in der nichtarchimedischen Funktionentheorie, Invent. Math. 2 (1967), 256-273.

Lut74 W. Lütkebohmert, Der Satz von Remmert-Stein in der nichtarchimedischen Funktionentheorie, Math. Z. 139 (1974), 69-84.

Mat80 H. Matsumura, Commutative algebra, second edition, Mathematics Lecture Note Series, vol. 56 (Benjamin/Cummings, Reading, MA, 1980).

OP97 J. Oesterlé and L. Pharamond dit d'Costa, Fermetures intégrales des $\overline{\mathbf{Z}}$-algèbres, J. Ramanujan Math. Soc. 12 (1997), 147-159.

Ray74 M. Raynaud, Géométrie analytique rigide d'après Tate, Kiehl, ..., in Table Ronde d'Analyse non archimédienne, Paris, 1972, Bulletin de la Société Mathématique de France, No. 39-40 (Société Mathématique de France, Paris, 1974), 319-327.

Siu69 Y.-T. Siu, Noetherianness of rings of holomorphic functions on Stein compact series, Proc. Amer. Math. Soc. 21 (1969), 483-489.

Jérôme Poineau jerome.poineau@univ-rennes1.fr

IRMAR, Université de Rennes 1, Campus de Beaulieu, 35042 Rennes cedex, France 